\title{
RESEARCH
}

Open Access

\section{Seasonal shifts in the gut microbiome indicate plastic responses to diet in wild geladas}

Alice Baniel ${ }^{1 *}$ (D), Katherine R. Amato2 ${ }^{2}$ Jacinta C. Beehner ${ }^{3,4}$, Thore J. Bergman ${ }^{3,5}$, Arianne Mercer ${ }^{6}$, Rachel F. Perlman ${ }^{7}$, Lauren Petrullo ${ }^{7}$, Laurie Reitsema ${ }^{8}$, Sierra Sams ${ }^{6}$, Amy Lu $^{1^{*+}+}$ and Noah Snyder-Mackler ${ }^{6,9,10,11^{*+}}$

\begin{abstract}
Background: Adaptive shifts in gut microbiome composition are one route by which animals adapt to seasonal changes in food availability and diet. However, outside of dietary shifts, other potential environmental drivers of gut microbial composition have rarely been investigated, particularly in organisms living in their natural environments.

Results: Here, we generated the largest wild nonhuman primate gut microbiome dataset to date to identify the environmental drivers of gut microbial diversity and function in 758 samples collected from wild Ethiopian geladas (Theropithecus gelada). Because geladas live in a cold, high-altitude environment and have a low-quality grass-based diet, they face extreme thermoregulatory and energetic constraints. We tested how proxies of food availability (rainfall) and thermoregulatory stress (temperature) predicted gut microbiome composition of geladas. The gelada gut microbiome composition covaried with rainfall and temperature in a pattern that suggests distinct responses to dietary and thermoregulatory challenges. Microbial changes were driven by differences in the main components of the diet across seasons: in rainier periods, the gut was dominated by cellulolytic/fermentative bacteria that specialized in digesting grass, while during dry periods the gut was dominated by bacteria that break down starches found in underground plant parts. Temperature had a comparatively smaller, but detectable, effect on the gut microbiome. During cold and dry periods, bacterial genes involved in energy, amino acid, and lipid metabolism increased, suggesting a stimulation of fermentation activity in the gut when thermoregulatory and nutritional stress co-occurred, and potentially helping geladas to maintain energy balance during challenging periods.

Conclusion: Together, these results shed light on the extent to which gut microbiota plasticity provides dietary and metabolic flexibility to the host, and might be a key factor to thriving in changing environments. On a longer evolutionary timescale, such metabolic flexibility provided by the gut microbiome may have also allowed members of Theropithecus to adopt a specialized diet, and colonize new high-altitude grassland habitats in East Africa.
\end{abstract}

Keywords: Gut microbiome, Graminivory, Seasonality, Thermoregulation, Theropithecus gelada, Primates

\footnotetext{
* Correspondence: alice.baniel@gmail.com; amy.lu@stonybrook.edu;

nsnyderm@asu.edu

${ }^{+}$Amy Lu and Noah Snyder-Mackler contributed equally to this work.

'Department of Anthropology, Stony Brook University, Stony Brook, NY

11794, USA

${ }^{6}$ Department of Psychology, University of Washington, Seattle, WA 98195,

USA

Full list of author information is available at the end of the article
}

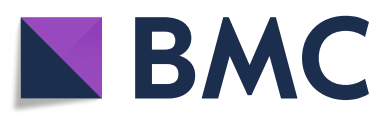

(- The Author(s). 2021 Open Access This article is licensed under a Creative Commons Attribution 4.0 International License, which permits use, sharing, adaptation, distribution and reproduction in any medium or format, as long as you give appropriate credit to the original author(s) and the source, provide a link to the Creative Commons licence, and indicate if changes were made. The images or other third party material in this article are included in the article's Creative Commons licence, unless indicated otherwise in a credit line to the material. If material is not included in the article's Creative Commons licence and your intended use is not permitted by statutory regulation or exceeds the permitted use, you will need to obtain permission directly from the copyright holder. To view a copy of this licence, visit http://creativecommons.org/licenses/by/4.0/ The Creative Commons Public Domain Dedication waiver (http://creativecommons.org/publicdomain/zero/1.0/) applies to the data made available in this article, unless otherwise stated in a credit line to the data. 


\section{Background}

Obtaining sufficient nutrients is a fundamental challenge for most animals. Yet, the availability and nutritional content of food can vary temporally and spatially in response to changes in climate and geography. Nutritional demands further vary in response to thermoregulatory needs and life history processes, such as growth and reproduction [1, 2]. Animals have evolved a variety of behavioral and physiological strategies to cope with these shifting demands, including altered feeding and activity patterns and increased mobilization of stored fat to fuel energetic demands [3-6]. Recently, the gut microbiome has been proposed as an additional avenue by which animals can cope with changing dietary landscapes and energetic challenges [7-9]. The gastrointestinal tract of animals harbors a dense microbial community that helps to break down and ferment plant structural carbohydrates, producing short-chain fatty acids (SCFAs) that can be used as an energy source by hosts [10-12]. The absorption of SCFAs in the gut may be particularly important for herbivorous species, such as foregut and hindgut fermenters, which obtain as much as $40-90 \%$ of their energy requirements from bacterial degradation of complex plant polysaccharides [1316]. Additionally, variation in gut microbiome composition affects the efficiency of caloric harvest and the metabolic programming of the host [11, 17-20]. For instance, in mice (Mus musculus) and humans, obese and lean individuals have strikingly different gut microbiota composition, with obese phenotypes being associated with higher energy extraction from diet and increased lipogenesis [21-23].

In wild mammals, the gut microbiome responds rapidly to seasonal and dietary changes [7, 24-29], presumably to buffer seasonal energetic challenges [7, 26]. For example, a simultaneous increase in bacterial taxa involved in fiber fermentation and in SCFA concentrations during the dry season may allow Mexican black howler monkeys (Alouatta pigra) to maintain energy balance during energetic shortfalls without changes in activity or ranging patterns [7]. Moreover, gut bacteria increase intestinal absorptive capacity, energy homeostasis, and fat burning during cold periods in mice [30], and improve digestive efficiency and SCFA production in energetically challenged ruminants living in cold environments and at highaltitude [31, 32]. These microbial shifts likely come at some cost. For instance, increases in microbes that improve host metabolism under certain conditions may reduce the abundance of microbes that support host immune function [33, 34]. However, in seasonal and nutritionally challenging environments, enduring these tradeoffs may be necessary for host survival and reproduction.

Geladas (Theropithecus gelada) represent an excellent system to investigate the relationship between gut microbiota composition and seasonal variation in host diet and energy needs. Despite being the only graminivorous primate with up to $90 \%$ of their diet comprised of grass [35, 36], their gastrointestinal tract appears poorly adapted to this specialization (but see [37, 38] for dental, manual, and locomotor adaptations), closely resembling their closest phylogenetic relatives, baboons (Papio spp.) - a taxon that is omnivorous [39]. To compensate, geladas may rely heavily on their gut microbiota to maximize nutrient extraction from grasses, likely through hindgut fermentation [39, 40]. Moreover, geladas live in a high-altitude, energetically demanding environment that exhibits marked inter- and intra-annual fluctuation in rainfall and temperature [35, 41]. During rainier months, when grass is abundant, they forage almost exclusively on aboveground graminoid leaves and seeds, and during drier months, when grass availability decreases, they shift heavily to underground foods (rhizomes, roots, corms, bulbs) $[35,42]$. This diet provides distinct challenges. Underground foods are considered a fallback food for geladas since they take additional time and effort to harvest, are harder to process, and are relied upon only when grasses are less abundant [35, 38]. Despite being considered a fallback food, these underground foods are rich in starches and carbohydrates, suggesting that they contain more nutritional energy than grass [43]. This high amount of energy, however, comes at some cost: roots and rhizomes are generally higher in fibers and lignin-and thus harder to digest than grasses. In addition to these nutritional challenges, ambient temperatures frequently drop to near freezing, and the metabolic costs of thermoregulation are known to strongly influence gelada physiology [44] and the timing of reproduction $[41,45]$. Thus, seasonal dietary shifts and temperature variation may lead to distinct digestive and thermoregulatory challenges.

One previous study on geladas from Guassa, Ethiopia, found that gut microbial communities of adult females shifted across seasons [40], supporting the hypothesis that the gut microbiome may help hosts confront environmental challenges. This study focused on adult females and assessed seasonal variation by separating the samples into two categorical seasons (i.e., rainy, dry). Our study expands on this study by including adult males, incorporating continuous climatic data across several years, and examining proxies of thermoregulatory stress (in addition to diet) as factors that can influence the composition and function of the gelada gut microbiome. Indeed, rainfall and temperature vary independently of each other and represent distinct ecological challenges in gelada ecosystems. Therefore, we were interested in further testing which aspect of gelada ecology more strongly determines seasonal microbiome shifts. 
We analyzed the gut microbiome composition and predicted microbiome function in 758 fecal samples across 5 years from 48 adult male and 86 adult female geladas living in the Ethiopian highlands in the Simien Mountains National Park. The Simien Mountains Gelada Research Project (SMGRP) has been collecting detailed climatologic, demographic, and behavioral data from this study population since 2006, allowing us to examine how ecological (rainfall and temperature) and individual (group membership, sex, reproductive status, and age) factors influence gelada gut microbiome composition. We hypothesized that ecological factors would be more strongly associated with variation in the gelada microbiome than individual factors, and that rainfall and temperature would have independent effects. In particular, we expected that rainfall, which is a good proxy for grass availability [35], would have the strongest effect on the gelada gut microbiome. We predicted that the taxonomic changes associated with rainfall would mainly reflect a shift to grass-based versus underground foodbased diet, in order to allow individuals to maximize energy extraction from those seasonal foods. We found that the gelada microbiome exhibited drastic shifts related to climatological variables; but individual variables, like age and sex, had minimal effects. Rainfall and temperature exerted independent effects on the microbial composition and predicted function-with rainfall having a stronger effect on the gelada gut microbiome. High rainfall, which is correlated with grass availability [35], was associated with more cellulolytic and fibrolytic bacterial taxa, when graminoid leaves were the main food source. Dry periods, when geladas consume more underground foods [35], were associated with more amylolytic and methanogenic taxa. Cold periods were further characterized by more amylolytic taxa, and hot periods by more methanogenic taxa. In both drier and colder periods, the gut microbiome shifted to predicted functions that suggested increased digestive efficiency, including energy, amino acid, and lipid metabolism. Overall, gelada gut microbial composition covaried with diet and temperature in a pattern that suggests plastic but distinct responses to dietary and metabolic challenges.

\section{Results}

The gelada gut microbiome

We identified 3295 amplicon sequence variants (ASVs) in 758 fecal samples (mean $\pm \mathrm{SD}=813 \pm 243$ ASVs per sample, range $=92-1730$ ) using deep $16 \mathrm{~S}$ rRNA gene amplicon sequencing. Most ASVs could be taxonomically assigned to the phylum (100\%), class (99\%), and order level (99\%), but assignments decreased substantially at the family $(85 \%)$ and genus (61\%) levels. These 3295 ASVs came from 16 different phyla, 65 families, and 200 genera (Table S1, Fig. 1, Figures S1-S2). Of the

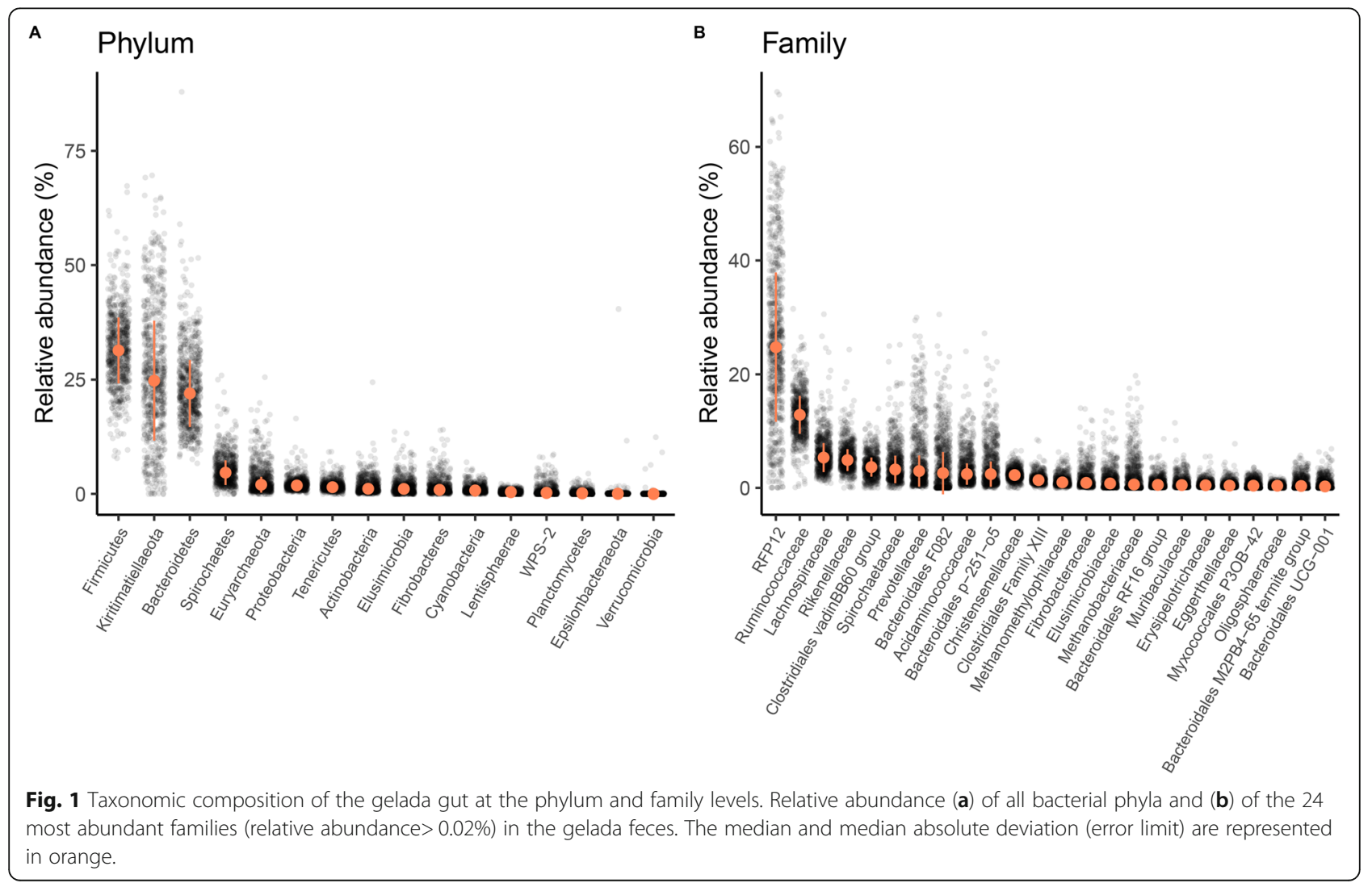


3295 ASVs, 170 (5\%) were present in at least 90\% of samples and form what can be considered the "core microbiota" of geladas (Table S2). The four most abundant bacterial phyla were Firmicutes (32\%), Kiritimatiellaeota (formerly called Verrucomicrobiota subdivision 5; 26\%), Bacteroidetes (23\%), and Spirochaetes (5\%) (Table S1, Fig. 1a). All microbes assigned to Kiritimatiellaeota were part of the RFP12 family and represent almost one quarter of the gelada gut microbiome (mean 26\%, range $0.02-70 \%$, Fig. 1b). Despite the abundance of RFP12, the 78 ASVs belonging to this group could not be assigned below the family level. We therefore queried these sequences against the NCBI database using BLAST $[46,47]$. These RFP12 sequences were most similar ( 97\%) to bacterial sequences previously found in the feces of hindgut herbivorous species (Asiatic elephant, domesticated horse, Somali wild ass, zebra, and black rhinoceros) [48] and, to a lesser extent, the rumen of several foregut fermenters (cattle, yak, red kangaroo; Table S3). Taken together, this indicates that the RFP12 family is a key microbe for herbivorous species, particularly hindgut fermenters, and likely plays an important fermentative role in plant digestion in geladas. Other taxa found at high frequency in the guts of ruminants and herbivorous hindgut fermenters were also prevalent in the gelada gut, including many cellulolytic/ fibrolytic (13\% Ruminococcaceae, 6\% Lachnospiraceae, 4\% Clostridiales vadinBB60 group, $1.5 \%$ Fibrobacteraceae) and fermentative families (5.3\% Rikenellaceae, 5\% Prevotellaceae, 4.1\% Bacteroidales F082) (Table S1, Fig. 1b and S1). The Spirochaetes phylum was mostly composed of Treponema (3.5\%), a genus involved in lignocellulose degradation [49].

\section{Dietary changes}

To examine how seasonal variation in rainfall and temperature was associated with changes in the gelada gut microbiome, we used measures of true climatic conditions, including monthly cumulative rainfall (an appropriate proxy of grass availability in the Simiens: [35]) and average monthly minimum temperature (a proxy of thermoregulatory constraint: [41, 44]). At the level of within-sample community diversity ("alpha diversity"), we found that cumulative rainfall was positively associated with Shannon evenness (Table 1, Fig. 2a, c) but had no effect on bacterial richness or Faith's phylogenetic diversity (Table S4, Figure S3). Thus, rainfall was associated with the relative abundance of ASVs within a sample but not the absolute number of ASVs or their phylogenetic diversity.

Cumulative rainfall significantly explained $3.3 \%$ of the overall compositional dissimilarity-or beta diversitybetween samples (as measured by Aitchison distance) (Table 2), which was less than that explained by two demographic variables: individual identity and unit (social group) membership (20\% and 6\%, respectively; Table 2). The first principal component of beta diversity, which explained $15 \%$ of variation, was strongly associated with rainfall $(r=0.43, t=12.93, \mathrm{df}=756, p<0.001$, Fig. $2 \mathrm{~b})$. The ASVs that loaded positively on PC1 (i.e., correlated with higher rainfall, Fig. 2d) were primarily from the families Prevotellaceae, Ruminococcaceae, and Lachnospiraceae (Table S5 and S6). By contrast, the ASVs that loaded negatively on PC1 (i.e., more abundant in low rainfall, Fig. 2d) belonged to the family RFP12 and a different subset of Ruminococcaceae that were not abundant during the wet season (Table S5 and S6).

Cumulative rainfall predicted the relative abundance of gut microbes at all taxonomic levels and was significantly associated with the relative abundance of $63 \%$ of bacterial families tested (59-81\% of taxa at other taxonomic levels, Fig. 3, Benjamini-Hochberg corrected $p$ values: $\left.p_{\mathrm{BH}}<0.05\right)$. Thus, across most taxa, there was a clear contrast in the relative abundance of gut bacteria between the wet and dry periods (Table S7, Fig. 4). In wetter periods, there was an increase in several important fermentative families from the Bacteroides order (including Prevotellaceae and Bacteroidaceae), as well as in several cellulolytic/fibrolytic taxa (Lachnospiraceae, Fibrobacteraceae, Spirochaetaceae, and several genera from the Ruminococcaceae; Figs. 4 and 5a), suggesting improved digestive efficiency of plant cell wall polysaccharides at a time when the gelada diet consists mainly of grasses. In particular, nine Prevotella genera as well as the Bacteroides genus were at higher abundance during wetter periods than drier periods (Table S7, Fig. 4b). There was also an increase in several proficient

Table 1 Determinants of alpha diversity, as measured by the Shannon index

\begin{tabular}{|c|c|c|c|c|c|}
\hline Fixed factor & Estimate & SE & 95\% confidence interval & LRT & $p$ value \\
\hline Sex (male) & -0.12 & 0.04 & {$[-0.20 ;-0.05]$} & 9.27 & 0.002 \\
\hline Age & 0.01 & 0.02 & {$[-0.02 ; 0.04]$} & 0.49 & 0.484 \\
\hline Cumulative rainfall & 0.05 & 0.02 & {$[0.02 ; 0.08]$} & 12.13 & $<0.001$ \\
\hline Minimum temperature & 0.00 & 0.01 & {$[-0.03 ; 0.03]$} & 0.04 & 0.848 \\
\hline Sequencing depth & 0.07 & 0.01 & {$[0.04 ; 0.10]$} & 24.24 & $<0.001$ \\
\hline
\end{tabular}

Parameters and tests are based on linear mixed models of 758 samples and 131 individuals, controlling for individual identity and unit membership. Factors with $p$ values less than 0.05 are highlighted in bold 


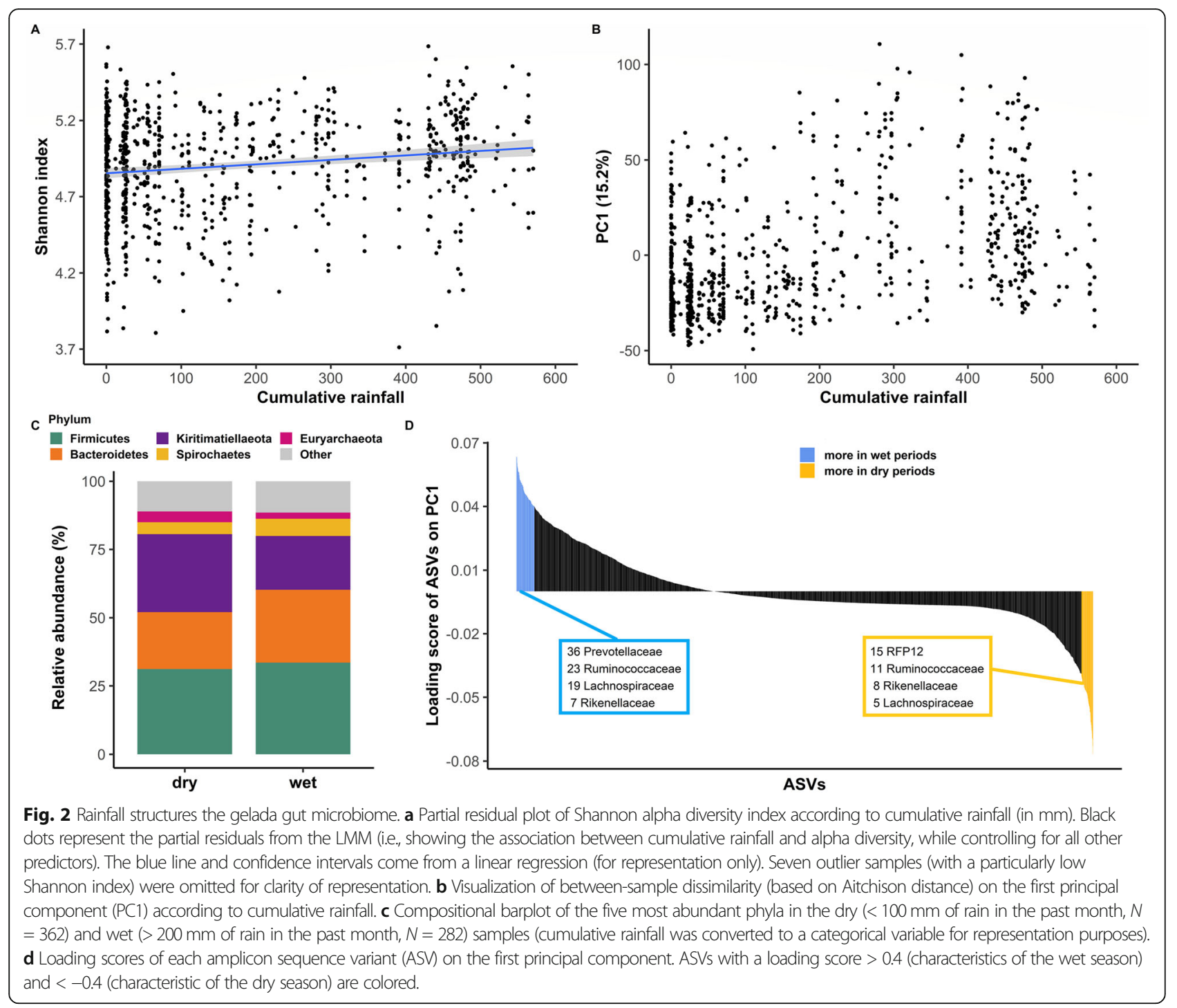

cellulolytic genera (e.g., Senegalimassilia, Butyrivibrio, Saccharofermentans, Cellulosilyticum, Marvinbryantia) (Table S7, Fig. 4b). By contrast, the dry season was characterized by an increase in amylolytic genera (Succinivibrio; Streptococcus and Pirellulaceae p-1088-a5 gut group), in several efficient sugar-fermenting families (Victivallales vadinBE97, Christensenellaceae), and in the methane-producer Methanobrevibacter, a genus known to increase the rate of fermentation and digestive efficiency (Table S7, and Figs. 4 and 5b). Consistent with our beta diversity analyses, we also found an increase in the relative abundance of the RFP12 family during the dry season (Table S7, Fig. 5b).

The taxonomic changes associated with rainfall also corresponded to changes in the predicted function of the gelada gut microbiome (as assessed by PICRUSt2: [50]). During wetter periods, functional changes tended to reflect the activity of the cellulolytic and fermentative bacterial taxa. Microbial pathways involved in the transport of molecules through bacterial membranes (e.g., ions, sugars, lipids, peptides), DNA replication and repair, and cell motility (Tables S8-S9, Figs. 6, S4 and $\mathrm{S} 5 \mathrm{~A}$ ) increased. We also found an increase in the metabolism of sugars (e.g., starch and sucrose metabolism, fructose, mannose, and galactose) (Figure S4 and S5A). Such activity probably reflects the exportation of sugarcleaving enzymes and cellulosome complex across the outer membrane $[12,51]$ of fibrolytic bacteria (complex polysaccharides are too big to penetrate directly inside bacteria and have to be cleaved first) and the absorption of the soluble oligosaccharides back across the bacterial membrane $[12,51]$.

During drier periods, the gelada gut harbored a greater abundance of bacterial genes involved in energy, amino acid, and lipid metabolism (Tables S8-S9 and Fig. 6a, c). In particular, cellular energy production and cellular 
Table 2 Determinants of beta diversity. Results of PERMANOVA testing for the predictors that significantly structure the gut microbiome of geladas, using 10,000 permutations and the Aitchison dissimilarity distance between samples. The $R$-squared values indicate the amount of between-sample variation explained by each variable.

\begin{tabular}{lll}
\hline Factor & $\boldsymbol{R}^{\mathbf{2}}(\%)$ & $\boldsymbol{p}$ value \\
\hline Individual $^{\mathrm{a}}$ & 20.25 & $<\mathbf{0 . 0 0 1}$ \\
Sequencing depth $^{\mathrm{b}}$ & 3.77 & $<\mathbf{0 . 0 0 1}$ \\
Unit $^{\mathrm{b}}$ & 5.84 & $<\mathbf{0 . 0 0 1}$ \\
Cumulative rainfall $^{\mathrm{b}}$ & 3.30 & $<\mathbf{0 . 0 0 1}$ \\
Minimum temperature $^{\mathrm{b}}$ & 0.33 & $<\mathbf{0 . 0 0 1}$ \\
Sex $^{\mathrm{b}}$ & 0.23 & $\mathbf{0 . 0 1 2}$ \\
Age $^{\mathrm{b}}$ & 0.19 & 0.045 \\
\hline
\end{tabular}

${ }^{a}$ We first fit a model with individual identity as the only predictor in a PERM ANOVA to estimate the sole effect of individual identity at explaining the overall gut composition of samples

${ }^{b}$ We then fit a second PERMANOVA model where all other predictors were fit, stratifying on individual identity to control for pseudoreplication of samples

from the same individual

activity were enhanced during this period, as evidenced by increases in pathways involved in the citric acid cycle, oxidative phosphorylation, and fatty acid synthesis and metabolism (Figure S4 and S5B). Other energy metabolism pathways also increased during drier periods, including the methane pathway and the carbon fixation pathways, which are important for generating energy in anaerobic bacteria (Figure S4 and S5B). Finally, drier periods were associated with an increase in functions related to the synthesis of proteinogenic amino acids (e.g., tryptophan), the translation and synthesis of proteins (Figure S4), and the synthesis of lipopolysaccharide.

Note, however, that the average weighted Nearest Sequence Taxon Index (NSTI) value across all samples (a measure of how similar bacteria from a sample are to reference genome sequences) was relatively high in our sample (mean $\pm \mathrm{SD}=0.60 \pm 0.13$ ) compared to other mammals [50]. Our results should therefore be interpreted with caution, in that they provide a general idea of the predicted gene family profiles based on bacteria that were functional characterized in previous studies.

\section{Temperature}

Compared to rainfall, minimum temperature had a much smaller impact on the gut microbiome. Average minimum temperature did not influence any metric of alpha diversity (Table 1 and S4, Figure S6A), and explained only $0.33 \%$ of the variation in beta diversity (Table 2, Figure S6B). Changes in temperature were significantly associated with the relative abundance of $5 \%$ of the families (5-22\% at other taxonomic levels; Fig. 3; $\left.p_{\mathrm{BH}}<0.05\right)$. More specifically, colder temperatures were characterized by a greater abundance of two amylolytic genera (Lactobacillus and Streptococcus); in several

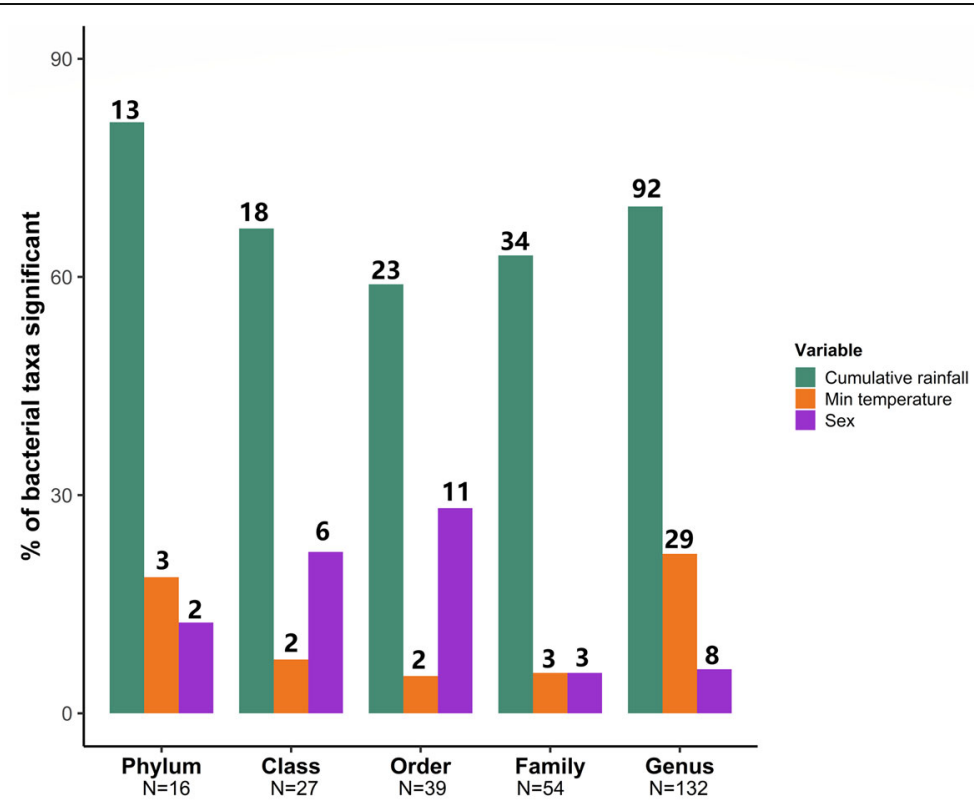

Fig. 3 Rainfall exerts the strongest effect on bacterial relative abundance. Percent of taxa that are significantly associated (Benjamini-Hochberg corrected $p$ values: $p_{\mathrm{BH}}<0.05$ ) with rainfall (purple bars), temperature (orange bars), or sex (green bars), across five taxonomic levels. For a given bacterial taxon, the significance of each predictor was assessed using a negative binomial GLMM of the count of this taxon per sample (controlling for sequencing depth as an offset factor, and including individual and unit membership as random effects). Only taxa with $p_{\mathrm{BH}}<0.05$ were considered significant. The numbers above the bars depict the number of taxa significantly differentially abundant, while the numbers below indicate the total taxa measured per level. Age was not significantly associated with relative abundance of any taxa at any level 

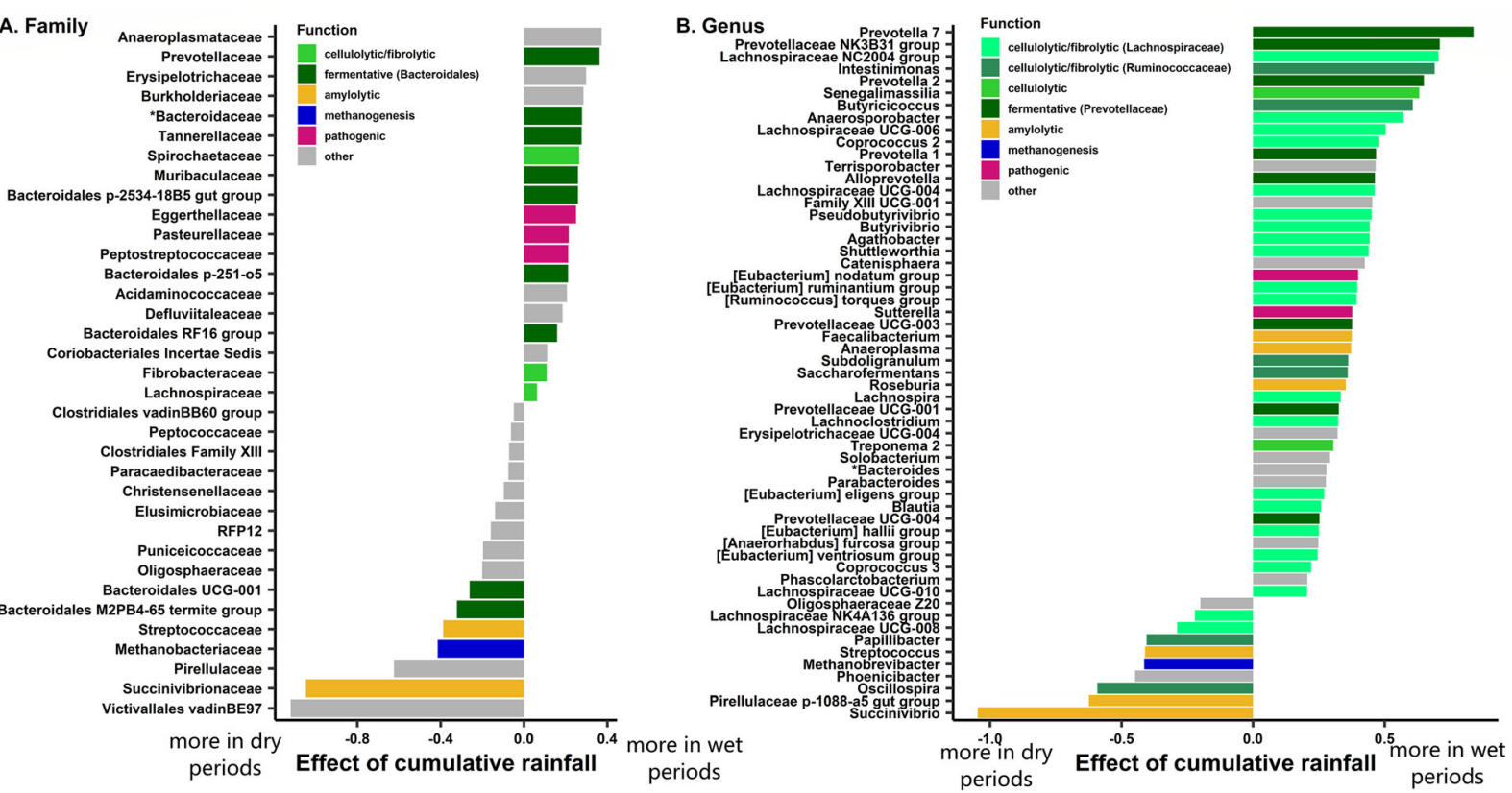

Fig. 4 Rainfall predicts the relative abundance of many bacterial taxa. a Families and $\mathbf{b}$ Genera that are found differentially abundant according to cumulative rainfall. The estimate of the cumulative rainfall effect for each taxon comes from a negative binomial GLMM modeled separately for the counts of each taxon across samples (controlling for sequencing depth as an offset factor, and including individual and unit membership as random effects). Taxa starting with "**" were fit with a binomial model instead. Only taxa with $p_{B H}<0.05$ were considered significant. For ease of representation on panel B, only genera with effect sizes $>|0.2|$ are represented. The full list of differentially abundant genera can be found in Table S7. Assignment of the "broad function" of a family or genus is for representation only, and is a simplification of the various functions subsumed within each taxonomic group.

sugar-fermenting (Hydrogenoanaerobacterium, Clostridium sensu stricto 1, Coprococcus 1) and cellulosedegrading bacteria (Marvinbryantia and two genera from the Ruminococcaceae family) (Table S7, Figure S7). By contrast, hotter temperatures were associated with an increase in Verrucomicrobia, in the methane-producer Methanobrevibacter, and in several cellulolytic/fibrolytic genera from the Ruminococcaceae and Lachnospiraceae families (Table S7, Figure S7).

Similar to our taxonomic analysis, we found that temperature had a much smaller effect on the predicted function of the gelada gut microbiome (Tables S8-S9, Figure S8). During colder periods, we found a predicted increase in bacterial pathways involved in lipid metabolism and energy production (notably in oxidative phosphorylation pathway; Figure S8). Other pathways that increased during colder periods involved DNA repair and recombination and the bacterial secretion system. During hotter weather, pathways were more poorly characterized and less specific, with predicted increases in methane metabolism and $\mathrm{ABC}$ transport (a membrane transporter).

\section{Sex, reproductive state, and age}

The gut microbiome of females exhibited higher alpha diversity compared to males, regardless of the metric (richness, evenness, and Shannon index) (Table 1 and
S4, Figure S9A). Across samples, however, sex explained little between-sample variation (i.e., $<1 \%$ ) (Table 2, Figure S9B). We detected only a handful of bacterial taxa that were differentially abundant according to sex (Table S7, Fig. 3). At the phylum level, females harbored more Verrucomicrobia and Proteobacteria (particularly from class Gammaproteobacteria, Deltaproteobacteria, and Alphaproteobacteria). At the family and genus levels, females had more taxa involved in lactic acid metabolism (Lactobacillaceae, Anaerovibrio), cellulolysis (Saccharofermentans), and regulation of glucose and fat transport (Erysipelatoclostridium). Males, on the other hand, only harbored more Pirellulales. No predicted metabolic pathway differed in abundance between males and females (Tables S8-S9).

Female reproductive state did not influence any alpha diversity metric (Table S10, Figure S10A) and was not a significant factor influencing beta diversity between samples (Table S11, Figure S10B). Very few taxa were differentially abundant according to female reproductive state (Table S12 and S13). Pregnant females harbored more Verrucomicrobiota (class Verrucomicrobiae) and Epsilonbacteraeota than cycling and lactating females (Table S13). In particular, the genus Helicobacter (within the family Epsiolonbacteroaeto) - a presumed pathogen-was highly prevalent in pregnant females (Table S13). No 

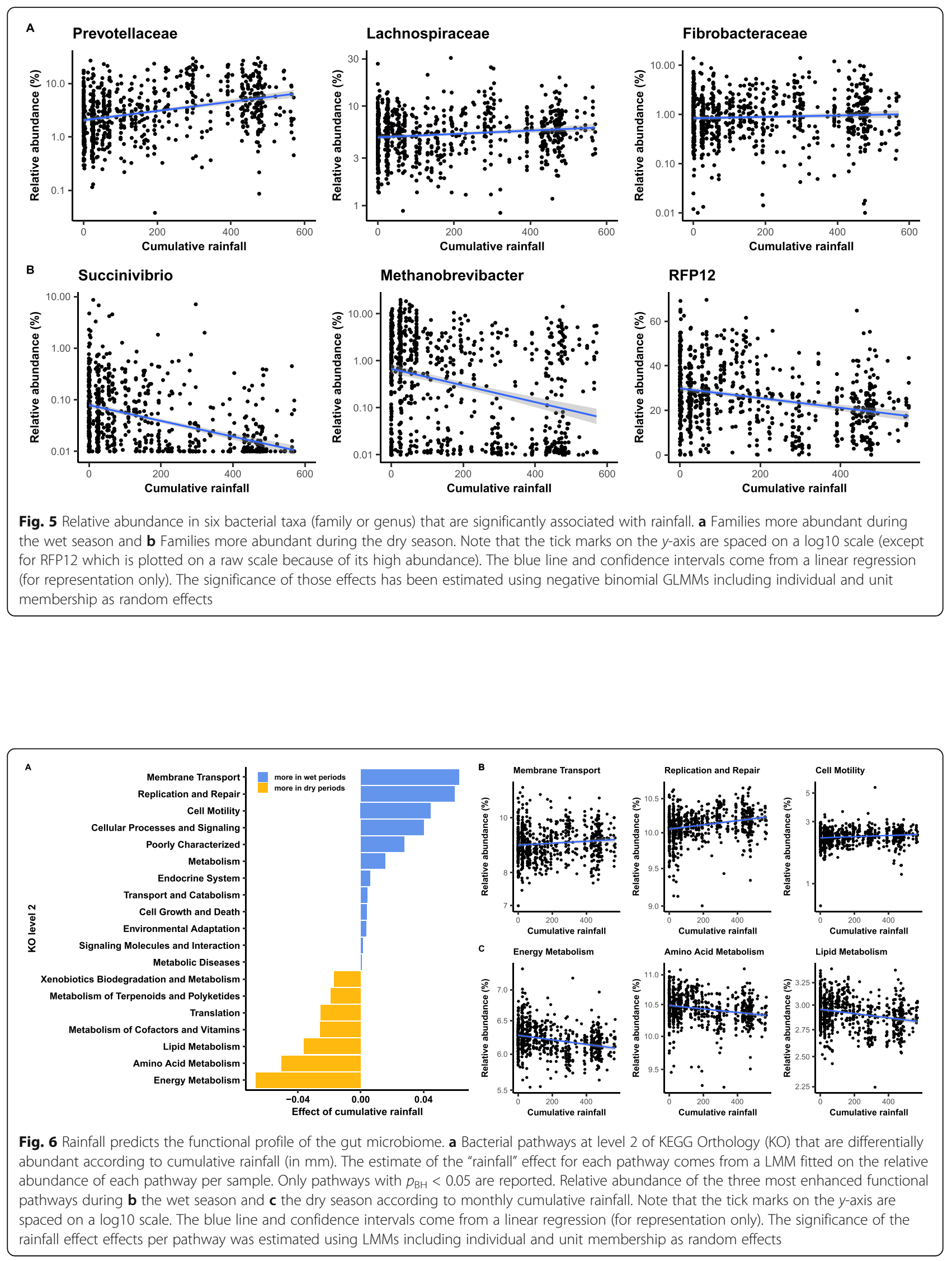
predicted metabolic pathways were found to differ based on reproductive state (Table S14-S15). Age did not influence any metric of alpha diversity (Table 1 and S4, Figure S11A) or beta diversity (Table 2, Figure S11B), and no bacterial taxa (Table S7) or predicted metabolic pathways (Table S8-S9) were differentially abundant between young and old adults.

\section{Discussion}

Our findings are consistent with the hypothesis that changes in the gelada gut microbiome may help animals cope with seasonal changes in food availability and thermoregulatory demands. First, the gelada gut microbiome was highly plastic and responded rapidly to seasonal fluctuations in climate-particularly rainfall (a proxy for available foods). Second, an increase in predicted bacterial functions involved in energy, amino acid, and lipid metabolism during both drier and colder periods suggested increased production of SCFAs, and more efficient digestion during energetically and thermoregulatory challenging periods. We further found that individual identity and social group explained nearly a third of the variation of the gelada microbiome, while other individual traits such as sex, reproductive state, and age had little effect on gut microbiome composition and function.

Rainfall was the strongest ecological factor influencing changes in the gelada gut microbiome, explaining 3.3\% of overall microbiome composition. In particular, cellulolytic/fibrolytic and fermentative bacterial taxa increased during wetter periods when grass, which is mostly composed of cellulose, was the primary food source, while amylolytic and methanogenic bacterial taxa increased during drier periods, when geladas incorporated more starch (i.e., amylose) and lignified food into their diet. This effect of rainfall on the gut microbiome was strong, despite the fact that geladas exhibit only moderate dietary changes across season (i.e., from only grass to less grass and more underground organs-but from the same plant species) compared to other mammals living in more seasonal environments, e.g., that switch from ripe fruits to more folivorous diets [7, 52, 53]. This pattern highlights the importance of the gut microbiome for geladas in processing their unique diet across seasons.

The efficiency of grass digestion in wet periods seems to rely on a syntropy between the first cellulolytic degraders (Ruminococcaceae, Lachnospiraceae, Fibrobacteraceae, Spirochaetes) and a high diversity of secondary fermenters (Prevotellaceae and Bacteroidales), which all increase in abundance during the wet season. The first degraders attach to the plant cell walls and hydrolyze cellulose, hemicellulose, and xylan into smaller polysaccharides and oligosaccharides [12,51], while secondary fermenters ferment those soluble polysaccharides into more simple sugars [10, 54]. Ruminococcaceae and Lachnospiraceae are the two main cellulolytic taxa in the mammalian gut and commonly increase in prevalence when animals eat more leaves and plants [7, 29]. In terms of secondary fermenters, Prevotella are widely known for their role in breaking down non-cellulosic polysaccharides and pectin $[10,12]$. They are the major constituent $(\sim 70 \%)$ of rumen bacteria [55], and commonly increase in high fiber or fruit diets $[29,53,56$, 57]. Members of Bacteroidales-and particularly from the Bacteroides genus-have some of the largest repertoires of carbohydrate degrading activities and are able to ferment a broad range of plant polysaccharides [10, 58-60]. The increase in these cellulolytic/fibrolytic taxa and the high versatility of the secondary fermenters likely allow geladas to optimally extract nutrients from grasses eaten during wet periods.

In contrast, during drier periods, when geladas relied more on underground storage organs, we found a corresponding increase in microbial families involved in amylolytic and saccharolytic activities (Succinivibrionaceae, Streptococcaceae, Christensenellaceae). Interestingly, Succinivibrionaceae also increased during periods of energetic stress in Tibetan macaques (Macaca thibetana) [26] and during the dry season in the Hazda hunter gatherers of Tanzania [61], suggesting that it might help hosts cope with diet-related energy shortfalls. The gelada microbiome during the dry season was also characterized by an increase in Methanobrevibacter, a genus containing hydrogenotrophic archaea that converts hydrogen and formate into methane [62]. The simultaneous enrichment of efficient hydrogen-producers (e.g., Christensenellaceae [63], Hydrogenoanaerobacterium: [64]) and formate-producers (Succinivibrionaceae: [65]), combined with methanogens during the dry season suggest that these taxa work together in syntropy to improve the efficiency of polysaccharide fermentation from starch in the gut in dry periods [66, 67]. In mice and humans, a higher abundance of methanogenic archaea increases calorie harvest from diet, facilitates SCFA production by other fermentative bacteria, and stimulates lipogenesis [66-69].

Finally, drier periods were also characterized by a large increase in the RFP 12 family (i.e., $~ 30 \%$ versus 18\% in wetter periods) from the Kiritimatiellaeota phylum. The RFP12 family remains poorly characterized but is increasingly recognized as being a keystone bacterial group in the hindgut of horses (Equus ferus caballus) [70-72], and a common inhabitant of the rumen of sheep (Ovis aries) or cattle [73-75]. The bacterial sequences belonging to the RFP12 family found in the gelada feces were, on average, $97 \%$ similar to bacterial sequences found in the feces of various hindgut herbivorous fermenters [48] 
and, to a lesser extent, in the rumen of foregut fermenters (Table S3). This suggests that the RFP12 family plays a particularly important, but thus far uncharacterized, digestive role for herbivorous species. In the case of geladas, it further suggests that it might be a keystone bacterial group for the digestion of some underground food components commonly eaten by the geladas during dry periods.

Overall, the gut microbes present in geladas are poorly represented by reference bacterial genomes in the two most common microbial databases: SILVA (updated 2017) [76] and Greengenes 13_8 (updated 2013) [77]. Taxonomic assignment of bacteria beyond the family level is relatively low (only 65\% of our reads were assigned to genus level) compared to assignment of sequences found in captive animals and humans. This poor taxonomic characterization limits our ability to understand the metabolic activities of gut microbiome communities, and highlights the need to generate more molecular microbial data from a wider range of nonmodel and wild organisms. The Kiritimatiellaeota phylum (and in particular the RFP12 family) would be a good candidate to focus on.

At the functional level, bacterial genes involved in energy, amino acid, and lipid metabolism increased in prevalence during the dry season. In particular, metabolic pathways linked to cellular respiration, methanogenesis, and carbon fixation pathways of prokaryotes became more common, strongly suggesting that both bacterial energy production and cellular activity were stimulated during this time. One interpretation of this data is that the increase in cellular activity simply reflects a dietary switch to starch, which is easier to hydrolyze than cellulose, and thus might more readily provoke a stimulation of bacterial activity and carbohydrate fermentation. Alternatively, the stimulation of bacterial energy metabolism and cellular activity could reflect a higher production of SCFAs by gut bacteria, supplying the host with additional energy in periods of nutrient restriction (when relying on fallback foods) [31, 78]. Similar increases in predicted bacterial energy metabolism have been found in energetically challenging environments (e.g., high altitude) in several other mammalian species and were correlated with higher SCFA production [31, 32]. Analysis of fecal SCFA profiles in geladas would help to identify if this is also the case in this high-altitude species. One caveat to our analysis is that the accuracy of the PICRUSt2 functional predictions may be lower than in other studies, especially when compared to published NSTI values in other mammals [50]. Previous studies of mammalian gut microbiomes conducted PICRUSt analyses on bacterial sequences grouped by OTUs, instead of ASVs. Because OTUs group sequences with $97 \%$ of similarity together, it removes $3 \%$ of genetic variation; and thus by definition OTUs will be closer to samples from reference genomes, potentially leading to lower NSTI values. Thus, although our analyses are reflecting only a limited portion of the bacterial community of geladas, it is not necessarily a worse representation than found in previous studies clustering bacteria by OTUs. Overall, while our predicted functions might give us a broad profile of the differences in metabolic activities of gut bacteria in the dry and wet seasons, they are not as precise as functional characterization from metagenomic or metabolomic data.

While it is clear that the gelada diet shifts during drier periods, it remains unknown if (and to what extent) geladas are nutritionally or energetically constrained during this time. Grass availability declines and geladas spend more time foraging and digging for underground plant parts during the dry season $[35,42]$. Such underground foods are usually considered fallback foods because individuals rely on them only when grass is less available and because they require long processing times [35, 38]. However, one study [42] found that geladas obtain just as much, or even more, calories from underground storage organs as they do from grass. Whether this increased caloric intake is offset by increased foraging costs is currently unknown. However, even if increased foraging costs were demonstrated, our data suggests that the gut microbiota may increase digestive efficiency from starchy food and thereby help geladas maintain or improve energetic status during the dry season. Future studies on seasonal changes in energy balance will help resolve this issue.

In contrast to the effect of rainfall, we found mixed evidence for the effect of temperature on the gut microbiome. Temperature only explained $\sim 0.33 \%$ of variation in the gelada gut microbiome composition. Furthermore, few taxa shifted in abundance between the coldest and hottest months, and most taxa affected by temperature were also affected by rainfall. This might be explained by the fact that rainfall (and thus diet) covaries with temperature to some extent (Pearson's correlation coefficient $=0.20)$ : geladas rely the most on underground foods in the hot-dry season (Feb. to May) and the most on grass on the cold-wet season (Jun. to Sep.) [35]. The cold-dry season (Oct. to Jan.), however, displays a mixed pattern of diet and temperature: grass availability is still high in Oct.-Nov. (following the rainy season) but decreases markedly in Dec.-Jan. [35, 41]. These 2 months are thus characterized by the introduction of underground foods in the diet and are also the coldest months of the year, making them likely the most challenging times for geladas (compounding nutritional and thermoregulatory challenges). Accordingly, cold periods were characterized by an increase in two amylolytic and 
lactate-producing taxa (Streptococcus, Lactobacillus), presumably to more efficiently extract starch from the underground foods. At the functional level, the energy and lipid metabolism of bacteria were also stimulated in the cold months, further suggesting some role of gut bacteria in stimulating host digestive efficiency and energy metabolism during thermoregulatory-demanding times.

These seasonal changes that increase energy production during colder periods may come at some cost. Such trade-offs have been proposed where shifts that benefit one aspect of host physiology consequently lead to a decrease in other microbes that may also be necessary for the host. For example, microbes that promote host digestive efficiency and energy metabolism may also promote inflammation or even suppress immune function [33, 79]. We did not detect any obvious evidence of these tradeoffs in geladas, but future work that incorporates detailed host immunological and functional microbial data is needed to help determine if such trade-offs exist.

Finally, the present study found that the gelada gut microbiome was largely explained by individual identity (20\%), a pattern consistent with data from a range of vertebrates [40, 80-82], including humans [83, 84]. However, the effect of social group was lower in geladas than reported for other social mammals (geladas: 6.0\% vs., e.g., yellow baboon, Papio cynocephalus: $18.6 \%$ of variation explained [85], black howler monkey: $14 \%$ [86], ring-tailed lemurs, Lemur catta: $21 \%$ [87], Welsh Mountain ponies: 14\%: [82]). The combination of large individual effects with weak social group effects closely resembles data reported for the Guassa gelada population [40], suggesting a general, but consistent pattern. The weak unit-level effects may result from the unique social system of geladas: Because social units often aggregate into large bands whose composition changes regularly, geladas may have a higher rate of inter-unit microbial transmission compared with other primates. Future studies should explore in more detail the intraindividual fluctuation in gut microbiome composition, and whether group differences in ranging patterns may explain these differences.

Other individual predictors, namely, age, sex, and female reproductive state, had a very limited effect on the gut microbiome, mirroring results in other mammals (yellow baboons: [28, 85], ring-tailed lemurs: [87], Verreaux's sifakas, Propithecus verreauxi: [29], chimpanzees, Pan troglodytes schweinfurthii: [88], rhesus monkeys, Macaca mulatta: [89], Welsh Mountain ponies [82], domestic dog, Canis lupus familiaris: [90], but see black howler monkeys: [34] or Egyptian fruit bats, Rousettus aegyptiacus: [80]). Although female geladas harbored higher microbial richness than males, this resulted in minimal differences in gut microbial composition and predicted function. Compared to males, females had higher abundance of Proteobacteria and Lactobacillus. These two bacterial taxa were previously reported to increase during pregnancy and lactation in humans and non-human primates [27, 91], and act as early colonizers of the infant gut [92-94]. Additionally, pregnant female geladas harbored more Helicobacter, a potentially pathogenic genus $[95,96]$. An increase in potentially pathogenic microbes in pregnant females was also observed in black howler monkeys [34] and was hypothesized to be the consequence of a trade-off between reproduction and immunity. These dynamics warrant further investigation.

Overall, the gut microbiome of geladas seems to be highly plastic and can respond rapidly to changes in host diet and thermoregulatory demands. Stimulation of bacteria cellular activity could allow geladas to maintain adequate or even improved energetic balance during dry and cold periods. Our study adds to an increasing body of literature suggesting that the gut microbiota is an important system providing dietary and metabolic flexibility for the host and might be a key factor influencing the acclimatization to changing environments $[8,48,97,98]$. In addition to fostering phenotypic plasticity, the gut microbiome is increasingly hypothesized to contribute to host evolution and speciation [97-99] given the strong host phylogenetic signal in mammalian microbiome composition and function $[100,101]$ and evidence of microbiome heritability [102-104]. To the extent that microbiomes affect host phenotypes under selection, they will also affect host evolutionary trajectories. In the case of geladas, a shift in gut microbiome composition was probably an important adaptive mechanism that allowed members of the Theropithecus genus to adopt a specialized dietary niche and diversify rapidly from Papio $\sim 5$ million years ago [105]. Contrary to host adaptive genetic mutations, which occur over the course of many generations, the gut microbiota can shift in response to changes in host diet in a matter of days [9]. Given that the common ancestor of Theropithecus and Papio was omnivorous [106, 107], dietary flexibility provided by the gut microbiome may have been an important first step allowing members of Theropithecus to exploit new grassland habitats in East Africa, leading to the evolution of a specialized diet and, ultimately, further genetic and phenotypic adaptation.

\section{Conclusion}

The gut microbiome of geladas is plastic and responds rapidly to changes in host diet and thermoregulatory demands. These shifts appear to help geladas cope with seasonal fluctuations in nutrient intake and energy homeostasis, potentially allowing them to maintain energy balance in challenging seasonal environments. Our 
study adds to an increasing body of literature suggesting that the gut microbiota is an important system that provides metabolic flexibility for the host, and is key to helping hosts adapt and shift to fluctuating environments. Future research in geladas and other animals will further uncover how the gut microbiota allows hosts to occupy unique dietary niches, potentially allowing host lineages to expand into new habitats, and facilitating speciation.

\section{Material and methods}

\section{Study population and fecal sample collection}

We collected fecal samples from a wild population of geladas living in the Simien Mountains National Park, in northern Ethiopia $\left(13^{\circ} 15^{\prime} \mathrm{N}, 38^{\circ} 00^{\prime} \mathrm{E}\right)$. Samples were collected over a 4-year period between Jan. 2015 and Feb. 2019. Geladas live in multi-level societies, where reproductive units (comprising a leader male, several adult females, their offspring, and occasionally 1-2 follower males) and bachelor groups (comprising between 1 and 10 adult males) form the smallest levels of the society that forage and sleep together in a "band" sharing the same home range [108]. Since Jan. 2006, the Simien Mountains Gelada Research Project (SMGRP) has collected demographic and behavioral data on over 200 individuals from two bands. All individuals are habituated to human observers on foot and are individually recognizable. Dates of birth of individuals were established using a combination of known $(N=42)$ and estimated $(N=89)$ birth dates. Estimated birth dates were calculated by using the mean individual age at major life-history milestones in our population (e.g., sexual maturation or first birth for females and canine eruption for males) $[109,110]$. Birth dates of unknown immigrant males were estimated using an established protocol based on body size and other age-related morphological characteristics [110]. Here, we focused only on samples from adult males and females. Adult males were included when they reached 7 years of age. At this age, males have reached adult body size in stature but not in weight $[110,111]$, and most males have dispersed into a non-natal group (i.e., $96 \%$ of our male samples, males could thus be leaders, followers, bachelors, or natals). Adult females were included after they had experienced their first sex skin swelling, a marker of reproductive maturation (which is around 4.65 years old in our population [109]).

Fecal samples of known adult and subadult male and female subjects were collected regularly and opportunistically during the study period. Immediately upon defecation, approximately $1.5 \mathrm{~g}$ of feces was collected in 3 $\mathrm{ml}$ of RNA later [112, 113], stored at room temperature for up to 2 months, and subsequently shipped to the University of Washington (UW). At UW, samples were stored at $-80^{\circ} \mathrm{C}$ until the sequencing libraries were prepared. A total of 758 samples (620 female samples, 138 male samples) were collected from 131 individuals (83 females, 48 males) (mean $\pm \mathrm{SD}=5.79 \pm 6.14$ samples per individual, range $=1-21)$ from 28 reproductive units and 4 bachelors groups (mean $\pm \mathrm{SD}=4.69 \pm 2.97$ number of individuals sampled per unit, range $=1-11$ ).

The reproductive state of females at the date of sample collection was assigned based on daily monitoring of individuals for the status of sex skin swellings and the birth of infants. We assigned the three reproductive states as follows: (1) Cycling began at the first sign of postpartum sex skin swelling and ended when a female conceived-with conception defined as 183 days (mean gestation length) before the birth of a subsequent infant [109]. (2) Pregnancy started on the date of conception and ended the day before parturition. (3) Finally, lactation started on the day of parturition and ended the day before the female's first postpartum swelling. Lactating females were further categorized as being in early lactation (infant $<1$ year old) or late lactation (infant $>1$ year old). When testing the effect of reproductive state, late lactating females were removed from the lactating category to include only females that were still nursing at the time of sample collection (females resume cycling when infants are $\sim 1.5$ years old in our population, which is presumably accompanied by infant weaning around the same time [109]). Furthermore, because pregnant females can abort their fetus during male takeover of their reproductive unit [114], some pregnancies might have been misidentified as cycling based on our method of back-calculating from the date of birth. We therefore removed cycling females that experienced a takeover in the previous 6 months before the date of sample collection ( $N=55$ samples) to avoid any misclassification of reproductive state in our analyses.

\section{Study site and climatic data}

The study area is located at $3200 \mathrm{~m}$ above sea level and is characterized as an Afroalpine grassland ecosystem, consisting of grassland plateaus, scrublands, and Ericaceous forests [115]. Fecal samples were collected across the year, with roughly equal coverage across seasons (244 in cold-dry, 298 in cold-wet, and 216 in hot-dry season as defined above). As part of the long-term monitoring of the SMGRP, daily cumulative rainfall and minimum and maximum temperature are recorded on a near-daily basis. We used the total cumulative rainfall over the 30 days prior to the date of fecal sample collection as a proxy for grass availability at the time of sample collection [35]. In addition, we used the average minimum daily temperatures in the 30 days preceding the date of sample collection as a proxy of thermoregulatory constraints. The average minimum temperature is less 
correlated with cumulative monthly rainfall than the average maximum temperature in the previous 30 days (correlation coefficient: 0.25 versus -0.56 ) and, more importantly, is more likely to reflect the physiological effect of thermoregulation on the body $[41,44]$.

\section{DNA extraction, sequencing, and data processing}

We prepared $16 \mathrm{~S}$ sequencing libraries using the protocols developed and optimized by the Earth Microbiome Project and the University of Minnesota Genomics Core (UMGC [116];). We extracted microbial DNA from the fecal samples using Qiagen's PowerLyzer PowerSoil DNA Isolation kit (Qiagen \#12855) following the standard protocol. We amplified the hypervariable V4 region of the 16S rRNA gene using PCR primer set $515 \mathrm{~F}$ (TCGTCGGCAGCGTCAGATGTGTATAAGAGACAG GTGYCAGCMGCCGCGGTAA) and 806R (GTCTCG TGGGCTCGGAGATGTGTATAAGAGACAGGGAC TACNVGGGTWTCTAA

T) from The Human Microbiome Project and a dualindexing approach [116]. Details of the amplification protocol can be accessed at https://smack-lab.com/ protocols/. The first PCR round aimed at amplifying the V4 region. Each $25 \mu \mathrm{l}$ PCR reaction well consisted of $12.5 \mu \mathrm{l}$ of Nebnext Ultra II Q5 mastermix, $1.0 \mu \mathrm{l}$ of each primer, and $25 \mathrm{ng}$ of total DNA in $10.5 \mu \mathrm{l}$ of nucleasefree water. PCR was performed in an Eppendorf thermocycler with a $100^{\circ} \mathrm{C}$ heated lid using the following cycling steps: an initial denaturing for $5 \mathrm{~min}$ at $95^{\circ} \mathrm{C}$; followed by 15 cycles of $20 \mathrm{~s}$ at $98^{\circ} \mathrm{C}, 15 \mathrm{~s}$ at $62^{\circ} \mathrm{C}, 60 \mathrm{~s}$ at $72{ }^{\circ} \mathrm{C}$, and a final hold at $4{ }^{\circ} \mathrm{C}$. We cleaned up the PCR reaction with a 2:1 ratio of SPRI beads to PCR amplified DNA. The second PCR round aimed at adding a unique index primer combination to molecularly barcode each sample. We took $4 \mu \mathrm{l}$ of product from the first PCR and added $6 \mu \mathrm{l}$ of Nebnext Ultra II Q5 mastermix and $1 \mu \mathrm{l}$ of $\mathrm{n} 5$ and $\mathrm{n} 7$ indexing primers, with each sample being assigned a unique $\mathrm{n} 5 / \mathrm{n} 7$ index primer combination. This $12 \mu \mathrm{l}$ reaction was placed in an Eppendorf thermocycler with a $100^{\circ} \mathrm{C}$ heated lid, denatured for 5 min at $95^{\circ} \mathrm{C}$, and amplified with 10 cycles of $20 \mathrm{~s}$ at $98^{\circ} \mathrm{C}, 15 \mathrm{~s}$ at $55^{\circ} \mathrm{C}$, and $60 \mathrm{~s}$ at $72^{\circ} \mathrm{C}$ with a final hold at $4{ }^{\circ} \mathrm{C}$. After a 2:1 SPRI bead clean-up, amplification of the $\mathrm{V} 4$ region was confirmed in a few samples using an AATI fragment analyzer, and all libraries were quantified using a qubit fluorometer. The libraries were then pooled in roughly equimolar amounts (each with their own unique indexing primer combination), spiked with $10 \%$ PhiX to increase library complexity, and sequenced together on a single Illumina NovaSeq 6000 SP 250 bp paired-end sequence flowcell.

We analyzed the resulting data using the Quantitative Insights Into Microbial Ecology 2 (QIIME2) platform $[117,118]$. After trimming low-quality bases from the de-multiplexed reads, we merged overlapping paired-end reads, and denoised the sequencing data by filtering and correcting Illumina amplicon sequencing errors using the Divisive Amplicon Denoising Algorithm 2 (DADA2: [119]) plugin incorporated in QIIME2. DADA2 infers sequences exactly resulting in amplicon sequence variants (ASVs). Forward and reverse reads were trimmed to 220 and 180 bases, respectively, to remove the low-quality portion of the sequences. The forward and reverse reads were then merged together and chimeric sequences were removed. Only samples with more than 20,000 reads were retained for analyses (following observation of rarefaction curves, Figure S12). After filtering, trimming, merging, and chimera removal, we retained a total of $348,390,395$ reads across the 758 fecal samples $(459,618$ $\pm 815,020$ reads per sample, range $=20,109-10,735,588$ ) . ASVs were taxonomically assigned using the q2-feature classifier in QIIME2 against version 132 of the SILVA database (updated December 2017) [76] based on 100\% similarity. Uninformative taxonomic assignments of ASVs found in SILVA (e.g., "wallaby metagenome," "unassigned bacteria," etc.) were converted to "NA" to simplify analyses at higher taxonomic levels. All ASVs belonging to the order WCHB1-41 (phylum Kiritimatiellaeota) were not assigned at the family level in the SILVA classification. However, in the Greengene classification (version 13_8) [77], all ASVs from this order in the gelada gut were assigned to the RFP12 family. Thus, we attribute the family RFP 12 to all ASVs from the order WCHB1-41 in SILVA classification. The 78 ASVs from the RFP12 family were found in particular high proportion in the gelada feces, but we did not have taxonomic information about those bacterial sequences below the family level. We thus mined previously published data to identify bacterial sequences most similar to the 78 RPF12 ASVs in geladas by using the NCBI BLAST search engine [46, 47]. For each of those 78 ASV, we searched for the most similar nucleotide sequences previously published, extracted its top five hits (i.e., most similar sequences based on the $E$ value), and summarized the distribution of those top hits per ASV across the 78 ASVs (including in which animal species those hits were found, see Table S3). The five top hits had an average of $97 \%$ nucleotide similarity (range, 93$100 \%$ ) with their respective similar gelada ASVs.

\section{Statistical analyses}

The count and taxonomy files generated by QIIME2 were imported into $\mathrm{R}$ version 3.5.2 [120] using the qiime2R package [121] and analyzed using the phyloseq package [122]. The majority of the 19,606 ASVs in our dataset were found at very low frequency or only in one sample (71\% of ASVs were found in only one sample and $6.2 \%$ of ASVs were not assigned at the phylum level). Thus, we 
further filtered the count table to retain only ASVs that had at least 500 reads in total in the dataset (i.e., $0.00014 \%$ relative abundance) to eliminate potentially artifactual sequences. With this filtering criteria, only 3295 ASVs remained, with all of them assigned at the phylum level and most (97\%) observed in at least two samples (Figure S13). The use of rarefaction (i.e., subsampling of the read count in each sample to a common sequencing depth) has been discouraged due to the loss of information and precision [123], as well as the use of count normalization methods from the RNA-seq field (e.g., DESeq2 or edgeR). However, microbiome datasets are more sparse (zero-inflated) and more asymmetrical than genetic expression datasets [124, 125]. Thus, we used a compositional approach when possible (e.g., centered-log-ratio normalization of the counts and Aitchison distance for beta diversity analysis) [125, 126], controlling for sample sequencing depth in multivariate analyses to account for repeated samples from the same individual.

We replicated alpha- and beta-diversity analyses using traditional rarefaction methods to facilitate comparisons with other studies. To generate the rarefied dataset, we randomly sampled 20,000 reads from the raw fastq files of each sample and processed this new rarefied dataset into the DADA2 pipeline. This dataset was further filtered to remove the low-frequency ASVs (i.e., ASVs not included in the pool of 3295 ASVs retained in the full dataset). This resulted in a dataset containing the same 758 samples, with 2853 ASVs and with relatively homogenous sequencing depth $(18205 \pm 1415$ reads per sample, range $=7460-19444)$. We rarefied reads from the raw fastq files prior to DADA2 processing instead of the traditional approach of rarefying after DADA2 using the rarefy_even_depth function of the phyloseq package [122] because the traditional approach did not eliminate the effect of sequencing depth (post-DADA2) on the alpha diversity metrics in the rarefied dataset. Subsampling the raw fastq files allowed us to better generate indices of alpha diversity per sample that did not depend on the total number of reads obtained initially for the sample.

All mixed models described below were run using either the lmer (for linear mixed models, LMMs) or glmer (for binomial and negative binomial generalized linear mixed models, GLMMs) functions of the lme4 package [127]. All quantitative variables (i.e., cumulative rainfall, averaged temperature, and age) were $\mathrm{z}$ transformed to have a mean of zero and a standard deviation of one to facilitate model convergence. The significance of the fixed factors was tested using a likelihood ratio test, LRT (assuming an asymptotic chi-square distribution of the test statistic) via the drop1 function. To test for significant pairwise differences between levels of multilevel categorical variables (i.e., reproductive state), post hoc Tukey's Honest Significant Difference tests were carried out using the multcomp package in $\mathrm{R}$ [128].

\section{Alpha-diversity analyses}

We calculated three measures of alpha diversity: observed richness (the total number of different ASVs in a sample), Shannon diversity index (accounts for both richness and evenness of ASVs in a sample), and Faith's phylogenetic diversity (accounts for phylogenetic distance between bacterial species, using the picante package [129]). We modeled each alpha diversity metric using linear mixed models: (i) as a function of age, sex, cumulative monthly rainfall, average monthly minimum temperature, and sequencing depth of the sample ( $N=758$ samples), and (ii) as a function of reproductive state (cycling, early lactating, and pregnant), age, cumulative monthly rainfall, and average monthly minimum temperature in samples collected from females $(N=439)$. Individual identity and unit membership were included as random effects to control for individual and unit repetition across samples. We also ran the same models on the rarefied dataset (Table S16).

\section{Beta-diversity analyses}

We then assessed how the same predictors were associated with between-sample community dissimilarity. To account for differences in sequencing depth between samples, the counts were normalized using the centeredlog-ratio (CLR) method (and using a pseudo count of 0.65 for zero counts) from the "compositions" package [130]. We then calculated the Aitchison distance between samples (i.e., simply the Euclidean distance between samples after clr transformation of the counts) [131] and conducted a Principal Component Analysis (PCA) (function "prcomp") to visually represent between-samples dissimilarity according to the predictors. This approach has been recommended for microbiome datasets [125] and allows for the projection of each sample onto individual principal components (PCS) and the variable loadings of ASVs onto each PC. While the first axis of variation correlated mostly with rainfall (Fig. 2b), the second PCA axis was correlated with sequencing depth and explained $11 \%$ of the variation (Figure S14). We used Permutational Multivariate Analysis of Variance (PERMANOVA) tests to assess the effect of the predictors on the Aitchison distance between samples (using 10,000 permutations and the "adonis2" function from the "vegan" package [132]). We ran three different models: (1) including all samples where we tested only the effect of individual identity and sequencing depth, (2) including all samples where we tested the effect of unit, age, sex, cumulative monthly rainfall, average monthly minimum temperature, and sequencing depth of the sample, and (3) including only female 
samples where we tested the effect of unit, reproductive state, age, cumulative monthly rainfall, and average monthly minimum temperature. In models 2 and 3, individual identity was included as a blocking factor ("strata") to control for repeated sampling. We also replicated beta diversity analysis on the rarefied dataset. We ran PERMANOVA tests using three complementary pairwise dissimilarity metrics (Bray-Curtis distance, unweighted and weighted UniFrac distances) to assess between-sample variation according to the same predictors (the same three models). Beta diversity results remained qualitatively similar (Table S17).

\section{Differential abundance testing}

We examined how our predictors were associated with differential abundance of bacteria (at the phylum, class, order, family, and genus levels) using negative binomial GLMMs. Compared to LMMs, negative binomial mixed models are better equipped to handle over-dispersed and zero-inflated distributions that often characterize microbiome datasets [133]. They also facilitate tests of several independent predictors while taking into account longitudinal designs including random effects. We first aggregated the counts (i.e., the number of reads per taxa and per sample) at the taxonomic level of interest. Only taxa that had an average relative abundance across samples $\geq 0.01 \%$ were tested. Then, for a given taxa, the count per sample was modeled as a function of (1) age, sex, cumulative monthly rainfall and averaged monthly minimum temperature (all samples), or (2) female reproductive state, age, cumulative monthly rainfall, and averaged monthly minimum temperature (female samples only). The log-transformed number of reads per sample was included as an offset term to control for variation in sequencing depth across samples. Individual identity and unit membership were included as random effects in all models. When negative binomial models failed to converge in some taxa, we converted the counts in presence/absence and modeled them with binomial GLMMs. Benjamini-Hochberg corrected p-values $<0.05$ were considered statistically significant.

\section{Functional profiling of microbiota}

We estimated the bacterial and archaeal genes present in the metagenomes of each sample using Phylogenetic Investigation of Communities by Reconstruction of Unobserved States version 2 (PICRUSt2) [50]. In brief, ASVs were aligned to reference sequences using HMME $\mathrm{R}$ [134] and placed into a reference tree using EPA-NG [135] and Gappa [136]. PICRUSt2 normalizes for multiple $16 \mathrm{~S}$ gene copies in bacteria using castor, a hidden state prediction tool [137]. The normalized data were used to predict gene family profiles, and mapped onto gene pathways using MinPath [138]. We followed the default protocols outlined on the PICRUSt2 GitHub page (https://github.com/picrust/picrust2/wiki). We investigated the predicted gene families using the Kyoto Encyclopedia of Genes and Genomes (KEGG) Orthology (KO) database. The accuracy of the PICRUSt2 predictions for each sample was assessed by calculating the weighted Nearest Sequence Taxon Index (NSTI) score, a measure of how similar the bacteria from the sample are to reference genome sequences. Five ASVs (out of 3295) had a NSTI score $>2$ and were removed from our final predictions. The association between the relative abundance of functional categories as estimated by PICRUSt2 and the predictors (on all samples or female samples only) were examined using LMMs. Only functional pathways that had $\geq 0.1 \%$ relative abundance across samples were tested. Individual identity and unit membership were included as random effects in all models.

\section{Supplementary Information}

The online version contains supplementary material available at https://doi. org/10.1186/s40168-020-00977-9.

\footnotetext{
Additional file 1: Table S1. Taxonomic composition of the gelada gut at the phylum, class, order, family and genus levels. The mean relative abundance and prevalence (\% of the samples with each taxon) of bacterial taxa are indicated. Table S2. Taxonomic composition of the core ASVs (i.e. present in at least $90 \%$ of samples) in the gelada gut at the order level. Table S3. Summary of the most similar bacterial sequences to the 78 ASVs belonging to the RFP12 family found in geladas feces. For each gelada RFP12 ASV, we searched the most similar nucleotide sequences found previously on NCBI BLAST, extracted its top five hits (i.e. most similar sequences based on Evalue) and summarized the distribution of those top hits across the 78 ASVs (identifying those animals in which similar sequences were found). Table S4. Predictors of observed richness and Faith's phylogenetic diversity (PD). Estimates with p-values $<0.05$ are highlighted in bold. Table S5. Loading scores of ASVs on the first principal component. Positive loadings correspond to wetter periods, while negative loadings correspond to drier periods. Table S6. Taxonomic distribution of seasonally differentially abundant taxa. Only taxa with a PC loading scores $>0.4$ and $<-0.4$ are included. Table S7. Differential abundance results for all taxa. Results were obtained by fitting negative binomial GLMMs for each taxa, controlling for individual identity and unit membership. The estimate and p-values of fixed effects are reported. Due to some negative binomial models that did not converge, taxa preceded by a "*" were modeled with a binomial GLMM, with presence/absence as the outcome variable. Table S8. Differential abundance results for KEGG pathways level 2. Model results were obtained by fitting LMMs on each pathway, while controlling for individual identity and unit membership. The estimate and p-value of the fixed effects are reported. Table S9. Differential abundance results for KEGG pathways level 3. Model results were obtained by fitting LMMs on each pathway, controlling for individual identity and unit membership. The estimate and pvalue of the fixed effects are reported. Table S10. Predictors of Shannon index, observed richness and Faith's phylogenetic diversity (PD) in females only. Estimates with p-values $<0.05$ are highlighted in bold. Table S11. Predictors of the structure of the female gelada gut microbiome. We carried out a PERMANOVA using 10,000 permutations and the Aitchison dissimilarity distance between samples. Table S12. Number of differentially abundant taxa for each predictor in the female samples. Table S13. Differential abundance results for female samples at five taxonomic levels. Table S14. Differential abundance results for KEGG pathways level 2 for female samples. Model results were obtained by fitting LMMs on each pathway, while controlling for individual identity and unit membership. The estimate and p-value of the fixed effects are reported. Table
} 
S15. Differential abundance results for KEGG pathways level 2 for female samples. Model results were obtained by fitting LMMs on each pathway, while controlling for individual identity and unit membership. The estimate and p-value of the fixed effects are reported. Table S16. Predictors of Shannon index, observed richness, and Faith's phylogenetic diversity (PD) on (1) all samples and (2) female samples on a rarefied dataset. Parameters and tests are based on 758/439 samples and 131/70 individuals in all models. The LMMs were performed controlling for individual identity and unit membership. The $95 \%$ confidence intervals that do not cross zero and p-values of statistically significant results are highlighted in bold. Table S17. Results of PERMANOVA testing for the effects that significantly structure the gut microbiome of geladas on a rarefied dataset for (1) all samples or (2) female samples only. We used Bray Curtis, unweighted Unifrac or weighted Unifrac distances to assess betweensample dissimilarity. 10000 permutations were carried out and individual identity was added as a strata in the model. The R-squared values indicate the amount of between-sample variation explained by each variable.

Additional file 2: Supplemental Figure 1. Taxonomic composition of the gelada gut microbiome at the phylum and family levels. Relative abundance (A) of all bacterial phyla and (B) of the 24 most abundant families (mean relative abundance $>0.02 \%$ ) in the gelada feces. The $y$-axis is on a $\log 10$ scale to better represent the variation across samples. The median and median absolute deviation (error limit) are represented in orange. Supplemental Figure 2. Genus composition of the gelada gut. Relative abundance of the 38 most abundant genera (mean relative abundance $>0.01 \%$ ) in the gelada feces. The tick marks on the $y$-axis are spaced on a $\log 10$ scale. The median and median absolute deviation (error limit) are represented in orange. Supplemental Figure 3. Rainfall is not associated with Observed richness and Faith's phylogenetic diversity. Partial residual plot of (A) Observed richness and (B) Faith's phylogenetic diversity (PD) according to cumulative rainfall (in $\mathrm{mm}$ ). Black dots represent the partial residuals from the LMM (i.e. showing the association between cumulative rainfall and alpha diversity, while controlling for all other predictors). The blue line and confidence intervals come from a linear regression (for representation only). One and 5 outlier samples (with a particularly low diversity) were omitted for panel $A$ and $B$ respectively for clarity of representation. Supplemental Figure 4. Bacterial functional pathways that significantly associated with cumulative rainfall at KO level 3. The estimate of the cumulative rainfall effect comes from a LMM fitted on the relative abundance of each pathway per sample. Only pathways with $\mathrm{p}_{\mathrm{BH}}<0.05$ were considered significant. For ease of representation, only pathways with effect sizes $>|0.006|$ are represented. The full list can be found in Table S9. Classification of $\mathrm{KO}$ level 3 pathways in broader cat egories were based on their KO level 2 assignment, with a few changes made for clarity of representation. Level 3 pathways from Metabolism of Other Amino Acids (level 2) were reclassified in the "Amino Acid Metabolism", Translation proteins and Replication, recombination and repair proteins (both level 3 and initially in Genetic Information Processing at level 2) were reclassified in "Transcription \& Translation" and "Replication and Repair" respectively. The category "Membrane Transport \& Cellular Signalling" regroups pathways from "Membrane Transport" and the other pathways from "Cellular Processes and Signaling". Supplemental Figure 5. Rainfall predicts the functional profile of the gut microbiome. Relative abundance of ten functional pathways (at KO level 3) that are enhanced (A) during the wet season and (B) during the dry season. Note that the tick marks on the $y$-axis are spaced on a log10 scale. The blue line and confidence intervals come from a linear regression (for representation only). The significance of the rainfall effect effects per pathway have been estimated using LMMs including individual and unit membership as random effects. Supplemental Figure $\mathbf{6}$. Small effect of ambient temperature on the gelada gut microbiome. (A) Partial residual plots of the three alpha diversity indices (Shannon index, Observed richness and Faith's phylogenetic diversity) according to the average minimum temperature in the previous month of sample collection (in ${ }^{\circ} \mathrm{C}$ ). Black dots represent the partial residuals from the LMM (i.e. showing the association between temperature and alpha diversity, while controlling for all other predictors). The blue line and confidence intervals come from a linear regression (for representation only). For clarity of representation, 9, 1, and 5 outlier samples (with a particularly low diversity) were omitted for Shannon, richness and Faith's PD, respectively. (B) Visualization of between-sample dissimilarity (based on Aitchison distance) on the first and second principal component according to minimum temperature. (C) Compositional barplot of the five most abundant phyla in the cold (i.e. $<8^{\circ} \mathrm{C}$ in the past month, $\left.\mathrm{N}=191\right)$ and hot $\left(>8^{\circ} \mathrm{C}\right.$ in the past month, $\mathrm{N}=567$ ) samples (minimum temperature was converted to a categorical variable for representation purposes). Supplemental Figure 7. Genera that significantly associated with average minimum temperature. The estimate for the effect of temperature for each taxa comes from a negative binomial GLMM controlling for sample sequencing depth as an offset factor, and including individual and unit membership as random effects.

Only taxa with $\mathrm{p}_{\mathrm{BH}}<0.05$ were considered significant. The full list can be found in Table S7. Supplemental Figure 8. Bacterial pathways that are differentially abundant according to average minimum temperature at $\mathrm{KO}$ (A) level 2 and (B) level 3. The estimate comes from a LMM fitted on the relative abundance of each pathway per sample. Only pathways with $\mathrm{p}_{\mathrm{BH}}<0.05$ were considered significant. For ease of representation on panel $B$, only pathways with effect size $>|0.002|$ were represented. The full list can be found in Table S8. Supplemental Figure 9. Effect of sex on the gelada gut microbiome. (A) Partial residual plots of the three alpha diversity indices (Shannon index, Observed richness and Faith's phylogenetic diversity) according to the sex of the sampled individual. Black dots represent partial residuals of the LMM. The median and median absolute deviation (error limit) of the distribution are represented in orange. Ten, 1 and 5 outlier samples (with a particularly low diversity) were omitted for Shannon, richness and Faith's PD respectively for clarity of representation. (B) Visualization of between-sample dissimilarity (based on Aitchison distance) on the first and second principal component according to sex. (C) Compositional barplot of the five most abundant phyla in male $(\mathrm{N}=138)$ and female $(\mathrm{N}=620)$ samples. Supplemental Figure 10. Effect of female reproductive state on the gelada gut microbiome. (A) Partial residual plots of the three alpha diversity indices (Shannon index, Observed richness and Faith's phylogenetic diversity) according to the reproductive state of the sampled female. Black dots represent partial residuals of the LMM. The median and median absolute deviation (error limit) of the distribution are represented in orange. Ten, 1 and 5 outlier samples (with a particularly low diversity) were omitted respectively for clarity of representation. (B) Visualization of betweensample dissimilarity (based on Aitchison distance) on the first and second principal component according to reproductive state. (C) Compositional barplot of the five most abundant phyla in pregnant $(\mathrm{N}=61)$, lactating ( $N=346)$ and cycling $(\mathrm{N}=158)$ female samples. Supplemental Figure 11. Effect of age on the gelada gut microbiome. (A) Partial residual plots of the three alpha diversity indices (Shannon index, Observed richness and Faith's phylogenetic diversity) according to the age of individuals at the date of sample collection (in years). Black dots represent the partial residuals from the GLMM (i.e. showing the association between age and alpha diversity, while controlling for all other predictors). The blue line and confidence intervals come from a linear regression (for representation only). Nine, 1 and 5 outlier samples (with a particularly low diversity) were omitted respectively for clarity of representation. (B) Visualization of betweensample dissimilarity (based on Aitchison distance) on the first and second principal component according to age. (C) Compositional barplot of the five most abundant phyla between young ( $<10$ years old, $\mathrm{N}=215 \mathrm{sam}$ ples), middle-aged (10 to 17 years old, $\mathrm{N}=420$ samples) and old (>17 years old, $\mathrm{N}=123$ samples) individuals (age was converted to a categorical variable for representation purposes only). Supplemental Figure 12. Rarefaction curves of samples. Only samples that had at least 20000 reads were included in this study. Supplemental Figure 13. $16 \mathrm{~S}$ sequencing and dataset characteristics. (A) Distribution of the total number of reads per sample (the tick marks on the $x$-axis are spaced on a log10 scale). (B) Distribution of the total number of ASVs per sample. Supplemental Fig-

ure 14. Visualization of differences in the gut microbiome composition according to sequencing depth of the samples based on Aitchison distance dissimilarity matrix. Points represent individual samples.

\section{Acknowledgements}

We thank the Ethiopian Wildlife Conservation Authority (EWCA), along with the wardens and staff of the Simien Mountains National Park for permission to conduct research and ongoing support to our long-term research project. We are also very grateful to the Simien Mountains Gelada Research Project 
field team for their help with field data collection, particularly our primary data collectors: Eshete Jejaw, Ambaye Fanta, Setey Girmay, Atirsaw Adugna, and Dereje Bewket. We would like to also thank Gavin M. Douglas for his expertise, troubleshooting, and discussion when using the PICRUSt2 pipeline. Special thanks to Johannes R. Björk and Elizabeth A. Archie for stimulating discussion about microbiome analyses and sharing code. Finally, we would also like to thank the two anonymous reviewers who provided useful comments.

\section{Authors' contributions}

Conceptualization, data curation, formal analysis, investigation, visualization: A.B, A.L., N.S.M; methodology: A.B., S.S., A.M., R.P.; writing-original draft: A.B, A.L., N.S.M; writing — review and editing: all authors; funding acquisition: A.L. N.S.M., J.C.B., T.J.B., L.R.; supervision: A.L. and N.S.M. The author(s) read and approved the final manuscript

\section{Funding}

This research was funded by the National Science Foundation (BCS-1723228, BCS-1723237, BCS-2010309, BCS-0715179, BCS-1732231, IOS-1255974, IOS1854359) and the L.S.B. Leakey Foundation (A.L.). The long term gelada research was supported by the University of Michigan, Stony Brook University, and Arizona State University, and the L.S.B. Leakey Foundation (A.L., J.C.B., T.J.B.). K.R.A. is supported as a fellow in the CIFAR 'Humans and the Microbiome' program. N.S.M. was supported by the National Institutes of Health (R00-AG051764).

\section{Availability of data and materials}

All 165 sequence data used in this study are available at the NCBI Sequence Read Archive (https://www.ncbi.nlm.nih.gov/) under BioProject ID PRJNA639843. Data and code (including how to run the QIIME2 pipeline on our data) are available at https://doi.org/10.5281/zenodo.3932310.

\section{Competing interests}

The authors declare no competing interests.

\section{Author details}

${ }^{1}$ Department of Anthropology, Stony Brook University, Stony Brook, NY 11794, USA. ²Department of Anthropology, Northwestern University, Evanston, IL 60208, USA. ${ }^{3}$ Department of Psychology, University of Michigan, Ann Arbor, MI 48109, USA. ${ }^{4}$ Department of Anthropology, University of Michigan, Ann Arbor, Ml 48109, USA. ${ }^{5}$ Department of Ecology and Evolutionary Biology, University of Michigan, Ann Arbor, Ml 48109, USA. ${ }^{6}$ Department of Psychology, University of Washington, Seattle, WA 98195, USA. ${ }^{7}$ Interdepartmental Doctoral Program in Anthropological Sciences, Stony Brook University, Stony Brook, NY 11794, USA. ${ }^{8}$ Department of Anthropology, University of Georgia, Athens, GA 30602, USA. ${ }^{9}$ Center for Evolution and Medicine, Arizona State University, Tempe, AZ 85281, USA. ${ }^{10}$ School of Life Sciences, Arizona State University, Tempe, AZ 85287, USA.

${ }^{11}$ Department of Biology, University of Washington, Seattle, WA 98195, USA.

Received: 16 July 2020 Accepted: 7 December 2020

Published online: 23 January 2021

\section{References}

1. Dufour DL, Sauther ML. Comparative and evolutionary dimensions of the energetics of human pregnancy and lactation. Am J Hum Biol. 2002;14:584602.

2. McNab BK. The physiological ecology of vertebrates: a view from energetics: Cornell University Press; Ithaca, NY. 2002.

3. van Schaik CP, Brockman DK. Seasonality in primate ecology, reproduction, and life history. Seasonality Primates. 2005:44:1.

4. Doran D. Influence of seasonality on activity patterns, feeding behavior, ranging, and grouping patterns in Tai chimpanzees. Int J Primatol. 1997;18: 183-206.

5. Gursky S. Effect of seasonality on the behavior of an insectivorous primate, Tarsius spectrum. Int J Primatol. 2000;21:477-95.

6. Dias PAD, Rangel-Negrín A, Canales-Espinosa D. Effects of lactation on the time-budgets and foraging patterns of female black howlers (Alouatta pigra). Am J Phys Anthropol. 2011;145:137-46.
7. Amato KR, Leigh SR, Kent A, Mackie RI, Yeoman CJ, Stumpf RM, et al. The gut microbiota appears to compensate for seasonal diet variation in the wild black howler monkey (Alouatta pigra). Microbial Ecol. 2015;69:434-43.

8. Candela M, Biagi E, Maccaferri S, Turroni S, Brigidi P. Intestinal microbiota is a plastic factor responding to environmental changes. Trends Microbiol. 2012;20:385-91.

9. David LA, Maurice CF, Carmody RN, Gootenberg DB, Button JE, Wolfe BE, et al. Diet rapidly and reproducibly alters the human gut microbiome. Nature. 2014:505:559-63.

10. Flint HJ, Scott KP, Duncan SH, Louis P, Forano E. Microbial degradation of complex carbohydrates in the gut. Gut Microbes. 2012;3:289-306.

11. Bäckhed F. Programming of host metabolism by the gut microbiota. Ann Nutr Metabol. 2011:58:44-52.

12. White BA, Lamed R, Bayer EA, Flint HJ. Biomass utilization by gut microbiomes. Annu Rev Microbiol. 2014:68:279-96.

13. Udén P, Rounsaville TR, Wiggans GR, Van Soest PJ. The measurement of liquid and solid digesta retention in ruminants, equines and rabbits given timothy (Phleum pratense) hay. Br J Nutr. 1982;48:329-39.

14. Bergman EN, Reid RS, Murray MG, Brockway JM, Whitelaw FG. Interconversions and production of volatile fatty acids in the sheep rumen. Biochem J. 1965:97:53-8.

15. Milton $\mathrm{K}, \mathrm{McBee} \mathrm{RH}$. Rates of fermentative digestion in the howler monkey, Alouatta palliata (primates: ceboidea). Comp Biochem Physiol A Comp Physiol. 1983;74:29-31.

16. Popovich DG, Jenkins DJ, Kendall CW, Dierenfeld ES, Carroll RW, Tariq N, et al. The western lowland gorilla diet has implications for the health of humans and other hominoids. J Nutr. 1997;127:2000-5.

17. De Filippo C, Cavalieri D, Di Paola M, Ramazzotti M, Poullet JB, Massart S, et al. Impact of diet in shaping gut microbiota revealed by a comparative study in children from Europe and rural Africa. Proc Natl Acad Sci U S A. 2010;107:14691-6

18. Krajmalnik-Brown R, Ihan Z-E, Kang D-W, DiBaise JK. Effects of gut microbes on nutrient absorption and energy regulation. Nutr Clin Pract. 2012;27:201-14.

19. Tremaroli $\vee$, Bäckhed F. Functional interactions between the gut microbiota and host metabolism. Nature. 2012;489:242-9.

20. Hanning I, Diaz-Sanchez S. The functionality of the gastrointestinal microbiome in non-human animals. Microbiome. 2015;3:51.

21. Turnbaugh PJ, Ley RE, Mahowald MA, Magrini V, Mardis ER, Gordon Jl. An obesity-associated gut microbiome with increased capacity for energy harvest. Nature. 2006:444:1027-31.

22. Turnbaugh PJ, Gordon J. The core gut microbiome, energy balance and obesity. J Physiol. 2009;457:480-4.

23. Tseng C-H, Wu C-Y. The gut microbiome in obesity. J Formos Med Assoc. 2019;118(Suppl 1):S3-9.

24. Maurice CF, Knowles SCL, Ladau J, Pollard KS, Fenton A, Pedersen AB, et al. Marked seasonal variation in the wild mouse gut microbiota. ISME J. 2015;9: 2423-34

25. Liu P-Y, Cheng A-C, Huang S-W, Chang H-W, Oshida T, Yu H-T. Variations in gut microbiota of Siberian flying squirrels correspond to seasonal phenological changes in their Hokkaido subarctic forest ecosystem. Microb Ecol. 2019;78:223-31

26. Sun B, Wang X, Bernstein S, Huffman MA, Xia D-P, Gu Z, et al. Marked variation between winter and spring gut microbiota in free-ranging Tibetan Macaques (Macaca thibetana). Sci Rep. 2016;6:26035.

27. Mallott EK, Amato KR. The microbial reproductive ecology of white-faced capuchins (Cebus capucinus). Am J Primatol. 2018;80:e22896.

28. Ren T, Grieneisen LE, Alberts SC, Archie EA, Wu M. Development, diet and dynamism: longitudinal and cross-sectional predictors of gut microbial communities in wild baboons. Environ Microbiol. 2016;18:1312-25.

29. Springer A, Fichtel C, Al-Ghalith GA, Koch F, Amato KR, Clayton JB, et al. Patterns of seasonality and group membership characterize the gut microbiota in a longitudinal study of wild Verreaux's sifakas (Propithecus verreauxi). Ecol Evol. 2017;7:5732-45

30. Chevalier C, Stojanović O, Colin DJ, Suarez-Zamorano N, Tarallo V, VeyratDurebex $C$, et al. Gut microbiota orchestrates energy homeostasis during cold. Cell. 2015;163:1360-74.

31. Zhang Z, Xu D, Wang L, Hao J, Wang J, Zhou X, et al. Convergent evolution of rumen microbiomes in high-altitude mammals. Curr Biol. 2016;26:1873-9.

32. Li H, Qu J, Li T, Wirth S, Zhang Y, Zhao X, et al. Diet simplification selects for high gut microbial diversity and strong fermenting ability in high-altitude pikas. Appl Microbiol Biotechnol. 2018;102:6739-51. 
33. Reese AT, Kearney SM. Incorporating functional trade-offs into studies of the gut microbiota. Curr Opin Microbiol. 2019;50:20-7.

34. Amato KR, Leigh SR, Kent A, Mackie RI, Yeoman CJ, Stumpf RM, et al. The role of gut microbes in satisfying the nutritional demands of adult and juvenile wild, black howler monkeys (Alouatta pigra). Am J Phys Anthropol. 2014;155:652-64

35. Jarvey JC, Low BS, Pappano DJ, Bergman TJ, Beehner JC. Graminivory and fallback foods: annual diet profile of geladas (Theropithecus gelada) living in the Simien Mountains National Park. Ethiopia. Int J Primatol. 2018;39:105-26.

36. Fashing PJ, Nguyen N, Venkataraman W, Kerby JT. Gelada feeding ecology in an intact ecosystem at Guassa, Ethiopia: variability over time and implications for theropith and hominin dietary evolution. Am J Phys Anthropol. 2014;155:1-16.

37. Wrangham RW. Bipedal locomotion as a feeding adaptation in gelada baboons, and its implications for hominid evolution. J Hum Evol. 1980;9: 329-31.

38. Venkataraman W, Glowacka H, Fritz J, Clauss M, Seyoum C, Nguyen N, et al. Effects of dietary fracture toughness and dental wear on chewing efficiency in geladas (Theropithecus gelada). Am J Phys Anthropol. 2014;155:17-32.

39. Mau M, Johann A, Sliwa A, Hummel J, Südekum K-H. Morphological and physiological aspects of digestive processes in the graminivorous primate Theropithecus gelada - a preliminary study. Am J Primatol. 2011;73:449-57.

40. Trosvik P, de Muinck EJ, Rueness EK, Fashing PJ, Beierschmitt EC, Callingham $\mathrm{KR}$, et al. Multilevel social structure and diet shape the gut microbiota of the gelada monkey, the only grazing primate. Microbiome. 2018;6:1-18.

41. Tinsley Johnson E, Snyder-Mackler N, Lu A, Bergman TJ, Beehner JC. Social and ecological drivers of reproductive seasonality in geladas. Behav Ecol. 2018:29:574-88.

42. Hunter CP. Ecological determinants of gelada ranging patterns (Theropithecus gelada): University of Liverpool; 2001. https://ethos.bl.uk/ OrderDetails.do?uin=uk.bl.ethos.250312

43. Dominy NJ, Vogel ER, Yeakel JD, Constantino P, Lucas PW. Mechanical properties of plant underground storage organs and implications for dietary models of early hominins. Evol Biol. 2008;35:159-75.

44. Beehner JC, McCann C. Seasonal and altitudinal effects on glucocorticoid metabolites in a wild primate (Theropithecus gelada). Physiol Behav. 2008;95: 508-14.

45. Carrera SC, Sen S, Heistermann M, Lu A, Beehner JC. Low rank and primiparity increase fecal glucocorticoid metabolites across gestation in wild geladas. Gen Comp Endocrinol. 2020;293:113494.

46. Altschul SF, Gish W, Miller W, Myers EW, Lipman DJ. Basic local alignment search tool. J Mol Biol. 1990;215:403-10.

47. Johnson M, Zaretskaya I, Raytselis Y, Merezhuk Y, McGinnis S, Madden TL. NCBI BLAST: a better web interface. Nucleic Acids Res. 2008;36(Web Server issue):W5-9.

48. Ley RE, Hamady M, Lozupone C, Turnbaugh PJ, Ramey RR, Bircher JS, et al. Evolution of mammals and their gut microbes. Science. 2008;320:1647-51.

49. Warnecke $F$, Luginbühl $P$, Ivanova $N$, Ghassemian M, Richardson $T H$, Stege $\mathrm{JT}$, et al. Metagenomic and functional analysis of hindgut microbiota of a wood-feeding higher termite. Nature. 2007:450:560-5.

50. Douglas GM, Maffei VJ, Zaneveld JR, Yurgel SN, Brown JR, Taylor CM, et al. PICRUSt2 for prediction of metagenome functions. Nat Biotechnol. 2020;38:685-8.

51. Biddle A, Stewart L, Blanchard J, Leschine S. Untangling the genetic basis of fibrolytic specialization by Lachnospiraceae and Ruminococcaceae in diverse gut communities. Diversity. 2013;5:627-40.

52. Hicks AL, Lee KJ, Couto-Rodriguez M, Patel J, Sinha R, Guo C, et al. Gut microbiomes of wild great apes fluctuate seasonally in response to diet. Nat Commun. 2018:9:1786.

53. Gomez A, Rothman JM, Petrzelkova K, Yeoman CJ, VIckova K, Umaña JD, et al. Temporal variation selects for diet-microbe co-metabolic traits in the gut of Gorilla spp. ISME J. 2016;10:514-26.

54. Flint HJ, Bayer EA, Rincon MT, Lamed R, White BA. Polysaccharide utilization by gut bacteria: potential for new insights from genomic analysis. Nat Rev Microbio. 2008:6:121-31.

55. van Gylswyk NO. Enumeration and presumptive identification of some functional groups of bacteria in the rumen of dairy cows fed grass silagebased diets. FEMS Microbiol Ecol. 1990;6:243-61.

56. Kovatcheva-Datchany P, Nilsson A, Akrami R, Lee YS, De Vadder F, Arora T, et al. Dietary fiber-induced improvement in glucose metabolism is associated with increased abundance of Prevotella. Cell Metab. 2015;22:971-82.
57. Rampelli S, Schnorr SL, Consolandi C, Turroni S, Severgnini M, Peano C, et al. Metagenome sequencing of the Hadza hunter-gatherer gut microbiota. Curr Biol. 2015;25:1682-93.

58. Comstock LE, Coyne MJ. Bacteroides thetaiotaomicron: a dynamic, nicheadapted human symbiont. BioEssays. 2003;25:926-9.

59. El Kaoutari A, Armougom F, Gordon Jl, Raoult D, Henrissat B. The abundance and variety of carbohydrate-active enzymes in the human gut microbiota. Nat Rev Microbiol. 2013;11:497-504.

60. Salyers AA, Vercellotti JR, West SE, Wilkins TD. Fermentation of mucin and plant polysaccharides by strains of Bacteroides from the human colon. Appl Environ Microbiol. 1977;33:319-22.

61. Smits SA, Leach J, Sonnenburg ED, Gonzalez CG, Lichtman JS, Reid G, et al. Seasonal cycling in the gut microbiome of the Hadza hunter-gatherers of Tanzania. Science. 2017;357:802-6.

62. Miller TL, Wolin MJ, Conway de Macario E, Macario AJ. Isolation of Methanobrevibacter smithii from human feces. Appl Environ Microbiol. 1982; 43:227-32.

63. Morotomi M, Nagai F, Watanabe Y. Description of Christensenella minuta gen. nov., sp. nov., isolated from human faeces, which forms a distinct branch in the order Clostridiales, and proposal of Christensenellaceae fam. nov. Int J Syst Evol Microbiol. 2012;62:144-9.

64. Song L, Dong X. Hydrogenoanaerobacterium saccharovorans gen. nov., sp. nov., isolated from H2-producing UASB granules. Int J Syst Evol Microbiol. 2009;59:295-9.

65. O'Herrin SM, Kenealy WR. Glucose and carbon dioxide metabolism by Succinivibrio dextrinosolvens. Appl Environ Microbiol. 1993;59:748-55.

66. Samuel BS, Gordon Jl. A humanized gnotobiotic mouse model of hostarchaeal-bacterial mutualism. Proceed Nat Acad Sci. 2006;103:10011-6.

67. Basseri RJ, Basseri B, Chong K, Youdim A, Low K, Hwang LJ, et al. W1367 Intestinal methane production in obese humans is associated with higher body mass index. Gastroenterology. 2010;138:S-708.

68. Zhang H, DiBaise JK, Zuccolo A, Kudrna D, Braidotti M, Yu Y, et al. Human gut microbiota in obesity and after gastric bypass. Proceed Nat Acad Sci. 2009;106:2365-70

69. Mathur R, Amichai M, Chua KS, Mirocha J, Barlow GM, Pimentel M. Methane and hydrogen positivity on breath test is associated with greater body mass index and body fat. J Clin Endocrinol Metabol. 2013;98:E698-702.

70. Steelman SM, Chowdhary BP, Dowd S, Suchodolski J, Janečka JE. Pyrosequencing of 165 rRNA genes in fecal samples reveals high diversity of hindgut microflora in horses and potential links to chronic laminitis. BMC Vet Res. 2012;8:231.

71. Costa MC, Silva G, Ramos RV, Staempfli HR, Arroyo LG, Kim P, et al. Characterization and comparison of the bacterial microbiota in different gastrointestinal tract compartments in horses. Vet J. 2015;205:74-80.

72. Edwards JE, Shetty SA, van den Berg P, Burden F, van Doorn DA, Pellikaan $W F$, et al. Multi-kingdom characterization of the core equine fecal microbiota based on multiple equine (sub)species. Animal Microbiome. 2020;2:6.

73. Ribeiro GO, Oss DB, He Z, Gruninger RJ, Elekwachi C, Forster RJ, et al. Repeated inoculation of cattle rumen with bison rumen contents alters the rumen microbiome and improves nitrogen digestibility in cattle. Sci Rep. 2017;7:1276

74. De Mulder T, Goossens K, Peiren N, Vandaele L, Haegeman A, De Tender C, et al. Exploring the methanogen and bacterial communities of rumen environments: solid adherent, fluid and epimural. FEMS Microbiol Ecol. 2017:93:fiw251.

75. Wang J, Fan H, Han Y, Zhao J, Zhou Z. Characterization of the microbial communities along the gastrointestinal tract of sheep by 454 pyrosequencing analysis. Asian Aust J Anim Sci. 2017;30:100-10.

76. Quast C, Pruesse E, Yilmaz P, Gerken J, Schweer T, Yarza P, et al. The SILVA ribosomal RNA gene database project: improved data processing and webbased tools. Nucleic Acids Res. 2013;41(Database issue):D590-6.

77. McDonald D, Price MN, Goodrich J, Nawrocki EP, DeSantis TZ, Probst A, et al. An improved Greengenes taxonomy with explicit ranks for ecological and evolutionary analyses of bacteria and archaea. ISME J. 2012;6:610-8.

78. Russell JB, Rychlik JL. Factors that alter rumen microbial ecology. Science. 2001;292:1119-22.

79. Vijendravarma RK, Narasimha S, Chakrabarti S, Babin A, Kolly S, Lemaitre B, et al. Gut physiology mediates a trade-off between adaptation to malnutrition and susceptibility to food-borne pathogens. Ecol Letters. 2015; 18:1078-86. 
80. Kolodny O, Weinberg M, Reshef L, Harten L, Hefetz A, Gophna U, et al. Coordinated change at the colony level in fruit bat fur microbiomes through time. Nat Ecol Evol. 2019;3:116-24.

81. Bik EM, Costello EK, Switzer AD, Callahan BJ, Holmes SP, Wells RS, et al Marine mammals harbor unique microbiotas shaped by and yet distinct from the sea. Nat Commun. 2016;7:10516.

82. Antwis RE, Lea JMD, Unwin B, Shultz S. Gut microbiome composition is associated with spatial structuring and social interactions in semi-feral Welsh Mountain ponies. Microbiome. 2018;6:207.

83. Costello EK, Lauber CL, Hamady M, Fierer N, Gordon Jl, Knight R. Bacterial community variation in human body habitats across space and time. Science. 2009;326:1694-7.

84. Human Microbiome Project Consortium. Structure, function and diversity of the healthy human microbiome. Nature. 2012;486:207-14.

85. Tung J, Barreiro LB, Burns MB, Grenier J-C, Lynch J, Grieneisen LE, et al. Social networks predict gut microbiome composition in wild baboons. Elife. 2015:4:e05224

86. Amato KR, Van Belle S, Di Fiore A, Estrada A, Stumpf R, White B, et al. Patterns in gut microbiota similarity associated with degree of sociality among sex classes of a neotropical primate. Microb Ecol. 2017;74:250-8.

87. Bennett G, Malone M, Sauther ML, Cuozzo FP, White B, Nelson KE, et al. Host age, social group, and habitat type influence the gut microbiota of wild ring-tailed lemurs (Lemur catta). Am J Primatol. 2016;78:883-92.

88. Degnan PH, Pusey AE, Lonsdorf EV, Goodall J, Wroblewski EE, Wilson ML, et al. Factors associated with the diversification of the gut microbial communities within chimpanzees from Gombe National Park. Proc Natl Acad Sci U S A. 2012;109:13034-9.

89. Adriansjach J, Baum ST, Lefkowitz EJ, Van Der Pol WJ, Buford TW, Colman RJ. Age-related differences in the gut microbiome of rhesus macaques. J Gerontol A Biol Sci Med Sci. 2020;75:1293-8.

90. Mizukami K, Uchiyama J, Igarashi H, Murakami H, Osumi T, Shima A, et al. Age-related analysis of the gut microbiome in a purebred dog colony. FEMS Microbiol Lett. 2019;366:fnz095.

91. Koren O, Goodrich JK, Cullender TC, Spor A, Laitinen K, Bäckhed HK, et al. Host remodeling of the gut microbiome and metabolic changes during pregnancy. Cell. 2012;150:470-80.

92. Shin N-R, Whon TW, Bae J-W. Proteobacteria: microbial signature of dysbiosis in gut microbiota. Trends Biotechnol. 2015;33:496-503.

93. Matsumiya Y, Kato N, Watanabe K, Kato H. Molecular epidemiological study of vertical transmission of vaginal Lactobacillus species from mothers to newborn infants in Japanese, by arbitrarily primed polymerase chain reaction. J Infect Chemother. 2002;8:43-9.

94. Martín R, Heilig GHJ, Zoetendal EG, Smidt H, Rodríguez JM. Diversity of the Lactobacillus group in breast milk and vagina of healthy women and potential role in the colonization of the infant gut. J Appl Microbiol. 2007; 103:2638-44.

95. Chichlowski M, Sharp JM, Vanderford DA, Myles MH, Hale LP. Helicobacter typhlonius and Helicobacter rodentium differentially affect the severity of colon inflammation and inflammation-associated neoplasia in IL 10-deficient mice. Comp Med. 2008;58:534-41.

96. Gao J-J, Zhang Y, Gerhard M, Mejias-Luque R, Zhang L, Vieth M, et al. Association between gut microbiota and Helicobacter pylori-related gastric lesions in a high-risk population of gastric cancer. Front Cell Infect Microbiol. 2018;8:202.

97. Alberdi A, Aizpurua O, Bohmann K, Zepeda-Mendoza ML, Gilbert MTP. Do vertebrate gut metagenomes confer rapid ecological adaptation? Trends Ecol Evol. 2016;31:689-99.

98. Macke E, Tasiemski A, Massol F, Callens M, Decaestecker E. Life history and eco-evolutionary dynamics in light of the gut microbiota. Oikos. 2017;126: 508-31.

99. Amato KR. Incorporating the gut microbiota into models of human and non-human primate ecology and evolution. Am J Phys Anthropol. 2016;159: $196-215$.

100. Groussin M, Mazel F, Sanders JG, Smillie CS, Lavergne S, Thuiller W, et al. Unraveling the processes shaping mammalian gut microbiomes over evolutionary time. Nat Commun. 2017;8:14319.

101. Amato KR, G Sanders J, Song SJ, Nute M, Metcalf JL, Thompson LR, et al. Evolutionary trends in host physiology outweigh dietary niche in structuring primate gut microbiomes. ISME J. 2019;13:576-87.

102. Goodrich JK, Waters JL, Poole AC, Sutter JL, Koren O, Blekhman R, et al. Human genetics shape the gut microbiome. Cell. 2014;159:789-99.
103. Blekhman R, Goodrich JK, Huang K, Sun Q, Bukowski R, Bell JT, et al. Host genetic variation impacts microbiome composition across human body sites. Genome Biol. 2015;16:191.

104. Waters $\mathrm{J}$, Ley RE. The human gut bacteria Christensenellaceae are widespread, heritable, and associated with health. BMC Biol. 2019;17:83.

105. Jablonski NG. Theropithecus: the rise and fall of a primate genus: Cambridge. Cambridge University Press; 2005.

106. Jolly CJ. The seed-eaters: a new model of hominid differentiation based on a baboon analogy. Man. 1970;5:5-26.

107. Dunbar RIM. Australopithecine diet based on a baboon analogy. J Hum Evol. 1976;5:161-7.

108. Snyder-Mackler N, Beehner JC, Bergman TJ. Defining higher levels in the multilevel societies of geladas (Theropithecus gelada). Int J Primatol. 2012;33: 1054-68.

109. Roberts EK, Lu A, Bergman TJ, Beehner JC. Female reproductive parameters in wild geladas (Theropithecus gelada). Int J Primatol. 2017;38:1-20.

110. Beehner JC, Gesquiere L, Seyfarth RM, Cheney DL, Alberts SC, Altmann J. Testosterone related to age and life-history stages in male baboons and geladas. Horm Behav. 2009;56:472-80.

111. Lu A, Bergman TJ, McCann C, Stinespring-Harris A, Beehner JC. Growth trajectories in wild geladas (Theropithecus gelada). Am J Primatol. 2016;78: 707-19.

112. Blekhman $R$, Tang $K$, Archie EA, Barreiro LB, Johnson ZP, Wilson ME, et al. Common methods for fecal sample storage in field studies yield consistent signatures of individual identity in microbiome sequencing data. Sci Rep. 2016;6.

113. Vlčková K, Mrázek J, Kopečný J, Petrželková KJ. Evaluation of different storage methods to characterize the fecal bacterial communities of captive western lowland gorillas (Gorilla gorilla gorilla). J Microbiol Methods. 2012;91:45-51.

114. Roberts EK, Lu A, Bergman TJ, Beehner JC. A Bruce effect in wild geladas. Science. 2012;335:1222-5.

115. Puff C, Nemomissa S. Plants of the Simen: a flora of the Simen Mountains and surroundings, northern Ethiopia. National Botanic Garden (Belgium); 2005.

116. Gohl DM, Vangay P, Garbe J, MacLean A, Hauge A, Becker A, et al. Systematic improvement of amplicon marker gene methods for increased accuracy in microbiome studies. Nat Biotechnol. 2016;34:942-9.

117. Hall M, Beiko RG. $16 S$ rRNA Gene analysis with QIIME2. Methods Mol Biol. 1849:2018:113-29.

118. Caporaso JG, Kuczynski J, Stombaugh J, Bittinger K, Bushman FD, Costello EK, et al. QIIME allows analysis of high-throughput community sequencing data. Nat Methods. 2010;7:335-6.

119. Callahan BJ, McMurdie PJ, Rosen MJ, Han AW, Johnson AJA, Holmes SP. DADA2: High-resolution sample inference from Illumina amplicon data. Nat Methods. 2016;13:581-3.

120. Team RC, Others. R: a language and environment for statistical computing. 2013.

121. Bisanz JE. qiime2R: importing QIIME2 artifacts and associated data into R sessions. Version 0 99; 2018. p. 13.

122. McMurdie PJ, Holmes S. phyloseq: an R package for reproducible interactive analysis and graphics of microbiome census data. PLoS One. 2013;8:e61217.

123. McMurdie PJ, Holmes S. Waste not, want not: why rarefying microbiome data is inadmissible. PLoS Comput Biol. 2014;10:e1003531.

124. Weiss S, Xu ZZ, Peddada S, Amir A, Bittinger K, Gonzalez A, et al. Normalization and microbial differential abundance strategies depend upon data characteristics. Microbiome. 2017:5:27.

125. Gloor GB, Macklaim JM, Pawlowsky-Glahn V, Egozcue JJ. Microbiome datasets are compositional: and this is not optional. Front Microbiol. 2017;8.

126. Gloor GB, Macklaim JM, Vu M, Fernandes AD. Compositional uncertainty should not be ignored in high-throughput sequencing data analysis. Aust J Stat. 2016;45:73

127. Bates D, Maechler M, Bolker B, Walker S. Ime4: Linear mixed-effects models using Eigen and S4. R package version 1.1-7; 2014

128. Hothorn T, Bretz F, Westfall P. Simultaneous inference in general parametric models. Biom J. 2008:50:346-63.

129. Kembel SW, Cowan PD, Helmus MR, Cornwell WK, Morlon H, Ackerly DD, et al. Picante: $\mathrm{R}$ tools for integrating phylogenies and ecology. Bioinformatics. 2010;26:1463-4.

130. van den Boogaart KG, Tolosana-Delgado R. "compositions": a unified R package to analyze compositional data. Comput Geosci. 2008;34:320-38.

131. Aitchison J, Barceló-Vidal C, Martín-Fernández JA, Pawlowsky-Glahn V. Logratio analysis and compositional distance. Math Geol. 2000;32:271-5. 
132. Oksanen J, Blanchet FG, Kindt R, Legendre P, O'hara RB, Simpson GL, et al. Vegan: community ecology package. R package version 1.17-4. URL http:// CRAN R-project org/package = vegan. 2010.

133. Zhang X, Pei Y-F, Zhang L, Guo B, Pendegraft AH, Zhuang W, et al. Negative binomial mixed models for analyzing longitudinal microbiome data. Front Microbiol. 2018;9.

134. Finn RD, Clements J, Eddy SR. HMMER web server: interactive sequence similarity searching. Nucleic Acids Res. 2011;39(suppl):W29-37.

135. Barbera P, Kozlov AM, Czech L, Morel B, Darriba D, Flouri T, et al. EPA-ng: massively parallel evolutionary placement of genetic sequences. Syst Biol. 2019;68:365-9

136. Czech L, Barbera P, Stamatakis A. Genesis and Gappa: library and toolkit for working with phylogenetic (placement) data. bioRxiv. 2019; https://www. biorxiv.org/content/10.1101/647958v1.abstract.

137. Louca S, Doebeli M. Efficient comparative phylogenetics on large trees. Bioinformatics. 2018;34:1053-5.

138. Ye Y, Doak TG. A parsimony approach to biological pathway reconstruction/ inference for genomes and metagenomes. PLoS Comput Biol. 2009;5: e1000465.

\section{Publisher's Note}

Springer Nature remains neutral with regard to jurisdictional claims in published maps and institutional affiliations.

Ready to submit your research? Choose BMC and benefit from:

- fast, convenient online submission

- thorough peer review by experienced researchers in your field

- rapid publication on acceptance

- support for research data, including large and complex data types

- gold Open Access which fosters wider collaboration and increased citations

- maximum visibility for your research: over $100 \mathrm{M}$ website views per year

At BMC, research is always in progress.

Learn more biomedcentral.com/submissions 